\title{
ON TUBULAR TILTING OBJECTS IN THE STABLE CATEGORY OF VECTOR BUNDLES
}

\author{
JIANMIN CHEN ${ }^{\dagger}$, YANAN LIN, AND SHIQUAN RUAN
}

\begin{abstract}
The present paper focuses on the study of the stable category of vector bundles for the weighted projective lines of weight triple. We find some important triangles in this category and use them to construct tilting objects with tubular endomorphism algebras for the case of genus one via cluster tilting theory.
\end{abstract}

\section{INTRODUCTION}

Weighted projective lines, introduced by Geigle and Lenzing, establish a link between many mathematical subjects such as representation theory of algebras [7], automorphic forms[13], and singularities[12]. Let $\mathbb{X}$ be a weighted projective line over an algebraically closed field $k$. By [7], the category of coherent sheaves on $\mathbb{X}$ is derived equivalent to the category of finite-dimensional modules over some canonical algebra, and the category of vector bundles on $\mathbb{X}$, as an additive category, is equivalent to the category of graded Cohen-Macaulay modules over its corresponding graded ring.

The present paper focuses on the study of a weighted projective line $\mathbb{X}$ of weight triple $\left(p_{1}, p_{2}, p_{3}\right)$. Kussin, Lenzing and Meltzer [12] proved that the category vect $\mathbb{X}$ of vector bundles on $\mathbb{X}$, under the distinguished exact structure, is a Frobenius category with the system $\mathcal{L}$ of all line bundles as the system of all indecomposable projective-injectives. Moreover, the attached stable category vect $\mathbb{X}=\operatorname{vect} \mathbb{X} /[\mathcal{L}]$ is triangulated. In particular, this triangulated category is closely related to the categories of finitely generated modules over Nakayama algebras, the stable categories of graded maximal Cohen-Macaulay modules and the singularity categories of some graded rings. So it is important and interesting to study the structure of this triangulated category, especially the triangles and tilting objects.

The excellent reference [12] is devoted to understanding the structure of vect $\mathbb{X}$. In particular, Kussin-Lenzing-Meltzer found a triangle consisting of rank-two bundles (see Section 2 for the notations)

$$
E_{L}\langle\vec{x}\rangle \rightarrow E_{L}\left\langle\vec{x}+\vec{x}_{i}\right\rangle \rightarrow E_{L}\left\langle\vec{x}-l_{i} \vec{x}_{i}\right\rangle\left(\left(l_{i}+1\right) \vec{x}_{i}\right) \rightarrow E_{L}\langle\vec{x}\rangle[1] .
$$

This triangle plays an important role in their construction of the tilting object

$$
T_{\text {cub }}=\bigoplus_{0 \leq \vec{x} \leq 2 \vec{\omega}+\vec{c}} E\langle\vec{x}\rangle,
$$

2010 Mathematics Subject Classification. 14A22, 14F05, 16G70, 16S99, 18E30.

Key words and phrases. tilting sheaf, weighted projective line, stable category, cluster category, tubular algebra.

$\dagger$ Corresponding author. 
whose endomorphism algebra is $k \vec{A}_{p_{1}-1} \otimes k \vec{A}_{p_{2}-1} \otimes k \vec{A}_{p_{3}-1}$. But there is still much unknown for this triangulated category. The aim of this paper is to find more triangles and tilting objects.

Notice by [16] that the stable category vect $\mathbb{X}$ is equivalent to the derived category of coherent sheaves on $\mathbb{X}$ if $\mathbb{X}$ is of genus one, that is, $\mathbb{X}$ is of weight type $(2,4,4),(2,3,6)$ or $(3,3,3)$. This implies that there exists a tilting object in vect $\mathbb{X}$ such that its endomorphism algebra is a canonical algebra of tubular type. We aim to construct such a tilting object (called tubular tilting object).

The main idea is to use cluster tilting theory. As we know that an advantage of cluster tilting theory over classical tilting theory is that there is an important tool, named cluster mutation, in cluster categories. Thus the usual procedure of going from a tilting object to another one by exchanging just one indecomposable direct summand gets more regular. We will construct the desired tilting object in vect $\mathbb{X}$ from a cluster tilting object in its cluster category.

We would like to emphasize that although there exists a tilting object $T_{\text {cub }}$ in the stable category vect $\mathbb{X}$, it is not clear whether $T_{\text {cub }}$ is a cluster tilting object in its cluster category. By studying the properties of tilting objects in the stable category, we show that $T_{\text {cub }}$ is a cluster tilting object for weight types $(2,4,4)$ and $(2,3,6)$, but not for weight type $(3,3,3)$. We construct a cluster tilting object for weight type $(3,3,3)$ as the original object for cluster mutation.

This paper is organized as follows: In Section 2, we recall some notions for later use. In Section 3, we present some triangles in the stable category of vector bundles on a weighted projective line of weight triple. The final section is devoted to studying tilting objects in the stable category. By using the cluster tilting mutations and triangles given in Section 3, we construct a tubular tilting object in the stable category of vector bundles for each weighted projective line of genus one and weight triple case by case. In view of the work on [4], which constructed a tubular tilting object in vect $\mathbb{X}$ of weight type $(2,2,2,2)$, we actually realize the construction of a tubular tilting object for all the weighted projective lines of genus one.

\section{Preliminaries}

In this section, we list some basic definitions concerning the weighted projective lines and cluster tilting theory. The properties we will use can be found in $[5,7,12]$.

2.1. The category of coherent sheaves $\operatorname{coh} \mathbb{X}$. Let $k$ be an algebraically closed field. A weighted projective line $\mathbb{X}$ over $k$ is specified by giving a collection $\lambda=$ $\left(\lambda_{1}, \lambda_{2}, \cdots, \lambda_{n}\right)$ of distinct points in the projective line $\mathbb{P}^{1}(k)$, and a weight sequence $p=\left(p_{1}, p_{2}, \cdots, p_{n}\right)$ of positive integers. The associated rank one abelian group $\mathbb{L}$ has generators $\vec{x}_{1}, \vec{x}_{2}, \cdots, \vec{x}_{n}$ with the relations $p_{1} \vec{x}_{1}=p_{2} \vec{x}_{2}=\cdots=p_{t} \vec{x}_{n}=: \vec{c}$. Each element $\vec{x} \in \mathbb{L}$ can be uniquely written in normal form

$$
\vec{x}=\sum_{i=1}^{n} l_{i} \vec{x}_{i}+l \vec{c}, \text { where } 0 \leq l_{i}<p_{i} \text { and } l \in \mathbb{Z} .
$$

The associated commutative algebra

$$
S=k\left[X_{1}, X_{2}, \cdots, X_{n}\right] /\left(f_{3}, \cdots, f_{n}\right):=k\left[x_{1}, x_{2}, \cdots, x_{n}\right],
$$

where $f_{i}=X_{i}^{p_{i}}-X_{2}^{p_{2}}+\lambda_{i} X_{1}^{p_{1}}, i=3, \cdots, n$, is $\mathbb{L}$-graded by $\operatorname{setting} \operatorname{deg}\left(x_{i}\right)=\vec{x}_{i}$. 
The category of coherent sheaves on $\mathbb{X}$ is the quotient of the category of finitely generated $\mathbb{L}$-graded $S$-modules over the Serre subcategory of finite length modules

$$
\operatorname{coh} \mathbb{X}:=\bmod ^{\mathbb{L}}(S) / \bmod _{0}^{\mathbb{L}}(S) .
$$

The free module $S$ gives the structure sheaf $\mathcal{O}$, and each line bundle is given by the grading shift $\mathcal{O}(\vec{x})$ for a uniquely determined element $\vec{x} \in \mathbb{L}$. Moreover, there is a natural isomorphism

$$
\operatorname{Hom}(\mathcal{O}(\vec{x}), \mathcal{O}(\vec{y}))=S_{\vec{y}-\vec{x}}
$$

The structure of $\operatorname{coh} \mathbb{X}$ was described in [7]. Especially, coh $\mathbb{X}$ is a hereditary abelian category with Serre duality of the form

$$
D \operatorname{Ext}^{1}(X, Y)=\operatorname{Hom}(Y, X(\vec{\omega})),
$$

where $\vec{\omega}=(n-2) \vec{c}-\sum_{i=1}^{n} \vec{x}_{i}$ is called the dualizing element of $\mathbb{L}$.

The Grothendieck group $K_{0}(\mathbb{X})$ of $\operatorname{coh} \mathbb{X}$ was computed by [7], and the definitions of homomorphism $\delta$, determinant map det, rank function rk, degree function deg, slope function $\mu$ can also be found in [7].

2.2. Stable category of vector bundle vect $\mathbb{X}$. We shall always assume that $\mathbb{X}$ is a weighted projective line of weight triple in the rest of the paper. Denote by vect $\mathbb{X}$ the full subcategory of $\operatorname{coh} \mathbb{X}$ formed by all vector bundles. A sequence

$$
0 \rightarrow X^{\prime} \rightarrow X \rightarrow X^{\prime \prime} \rightarrow 0
$$

in vect $\mathbb{X}$ is called distinguished exact if for each line bundle $L$ the induced sequence

$$
0 \rightarrow \operatorname{Hom}\left(L, X^{\prime}\right) \rightarrow \operatorname{Hom}(L, X) \rightarrow \operatorname{Hom}\left(L, X^{\prime \prime}\right) \rightarrow 0
$$

is exact. The distinguished exact sequences define a Frobenius exact structure on vect $\mathbb{X}$ such that the system of all line bundles is the system of all indecomposable projective-injectives. By a general result of [8], the related stable category vect $\mathbb{X}=$ vect $\mathbb{X} /[\mathcal{L}]$ is a triangulated category. For simplicity of notations, in the rest of the paper we denote the stable category vect $\mathbb{X}$ by $\mathscr{D}$ and denote the homomorphism space between $X$ and $Y$ in $\mathscr{D}$ by $\mathscr{D}(X, Y)$.

By [12], $\mathscr{D}$ is a Hom-finite, homologically finite, Krull-Schmidt category with Serre duality: $\mathscr{D}(X, Y[1])=D \mathscr{D}(Y, X(\vec{\omega}))$ for any two objects $X$ and $Y$ in $\mathscr{D}$. For a line bundle $L$ and $0 \leq \vec{x} \leq 2 \vec{\omega}+\vec{c}=\sum_{i=1}^{3}\left(p_{i}-2\right) \vec{x}_{i}$, the middle term of the non-split exact sequence

$$
0 \rightarrow L(\vec{\omega}) \rightarrow \bullet \rightarrow L(\vec{x}) \rightarrow 0
$$

in vect $\mathbb{X}$ is unique up to isomorphism. We call it the extension bundle determined by $(L, \vec{x})$, and denote it by $E_{L}\langle\vec{x}\rangle$. We simply denote by $E_{L}:=E_{L}\langle 0\rangle$ and call it the Auslander bundle associate with $L$, denote by $E\langle\vec{x}\rangle:=E_{\mathcal{O}}\langle\vec{x}\rangle$. In particular, $E:=E_{\mathcal{O}}\langle 0\rangle$.

Details about the structure of $\mathscr{D}$, the injective hull, the projective cover, the suspension for extension bundles, and homomorphism spaces between extension bundles are given in [12]. Based on the work of [12], Lenzing and the third-named author [15] further studied extension bundles in vect $\mathbb{X}$, and obtained the following basic property. For the convenience of the readers, we sketch the proof. 
Lemma 2.1. Assume $\vec{x}, \vec{y}, \vec{z} \in \mathbb{L}, \vec{x}=\sum_{i=1}^{3} l_{i} \vec{x}_{i}$ with $0 \leq l_{i} \leq p_{i}-2$ for $i=1,2,3$.

Then $E\langle\vec{x}\rangle=E\langle\vec{y}\rangle(\vec{z})$ if and only if one of the following conditions holds

- $\vec{y}=\vec{x}$ and $\vec{z}=0$

- $\vec{y}=l_{j} \vec{x}_{j}+\sum_{i \neq j}\left(p_{i}-2-l_{i}\right) \vec{x}_{i}$ and $\vec{z}=\sum_{i \neq j}\left(l_{i}+1\right) \vec{x}_{i}-\vec{c}$ for some $1 \leq j \leq 3$.

Proof Assume that $\vec{y}=\sum_{i=1}^{3} k_{i} \vec{x}_{i}$ and $\vec{z}=\sum_{i=1}^{3} \lambda_{i} \vec{x}_{i}+\lambda \vec{c}$ are both in normal forms. Then $E\langle\vec{x}\rangle=E\langle\vec{y}\rangle(\vec{z})$ if and only if they have the same class in the Grothendieck group since they are both exceptional in coh $\mathbb{X}([12]$, Theorem 4.2), hence we get

$$
[\mathcal{O}(\vec{w})]+[\mathcal{O}(\vec{x})]=[\mathcal{O}(\vec{w}+\vec{z})]+[\mathcal{O}(\vec{y}+\vec{z})],
$$

that is,

$$
\begin{aligned}
& {\left[\mathcal{O}\left(\sum_{i=1}^{3}\left(p_{i}-1\right) \vec{x}_{i}-2 \vec{c}\right)\right]+\left[\mathcal{O}\left(\sum_{i=1}^{3} l_{i} \vec{x}_{i}\right)\right] } \\
= & {\left[\mathcal{O}\left(\sum_{i=1}^{3}\left(\lambda_{i}-1\right) \vec{x}_{i}+(\lambda+1) \vec{c}\right)\right]+\left[\mathcal{O}\left(\sum_{i=1}^{3}\left(k_{i}+\lambda_{i}\right) \vec{x}_{i}+\lambda \vec{c}\right)\right] . }
\end{aligned}
$$

Representing each summand of (2.1) by the basis $\{[\mathcal{O}(\vec{x})] \mid 0 \leq \vec{x} \leq \vec{c}\}$ and comparing the determinants in both sides, we obtain the result.

Recall that an object $T$ in $\mathscr{D}$ is called tilting if

- $T$ is extension-free, i.e., $\mathscr{D}(T, T[n])=0$ for each non-zero integer $n$.

- $T$ generates the triangulated category $\mathscr{D}$, i.e., the smallest thick triangulated subcategory $\langle T\rangle$ containing $T$ is $\mathscr{D}$.

It is not easy to construct tilting objects in the stable category since all the line bundles are killed in $\mathscr{D}$ and the minimal rank of the objects in $\mathscr{D}$ is two. By investigating homomorphism spaces and important triangles for rank-two bundles in $\mathscr{D}$, Kussin-Lenzing-Meltzer finally obtained a tilting object consisting of rank-two bundles as follows:

Lemma 2.2 (Tilting cuboid, [12]). Let L be a line bundle. Then

$$
T_{\text {cub }}(L)=\bigoplus_{0 \leq \vec{x} \leq 2 \vec{\omega}+\vec{c}} E_{L}\langle\vec{x}\rangle
$$

is a tilting object in $\mathscr{D}$ with endomorphism ring

$$
\underline{\operatorname{End}}\left(T_{\text {cub }}(L)\right) \cong k \vec{A}_{p_{1}-1} \otimes k \vec{A}_{p_{2}-1} \otimes k \vec{A}_{p_{3}-1} .
$$

We denote by $T_{\text {cub }}=T_{\text {cub }}(\mathcal{O})$ in the rest of paper.

2.3. Relationship to cluster tilting theory. We assume $\mathbb{X}$ is a weighted projective line $\mathbb{X}$ of genus one and weight triple in this subsection, that is, $\mathbb{X}$ is of weight type $(2,3,6),(2,4,4)$ or $(3,3,3)$. We have Riemann-Roch Formula [14], the tubular factorization property and a bijective, monotonous map $\alpha: \mathbb{Q} \rightarrow \mathbb{Q}$ with $\alpha(q)>q$ for all $q \in \mathbb{Q}$ such that $\mu(X[1])=\alpha(\mu(X))$ for each indecomposable vector bundle $X$. Precisely, $\alpha^{-1}(0)=-\frac{3}{2}$ for weight type $(3,3,3), \alpha^{-1}(0)=-2$ for weight type $(2,4,4)$ and $\alpha^{-1}(0)=-3$ for weight type $(2,3,6)$ ([12], Theorem A.3). For any $a \in \mathbb{Q}$, the interval category $\mathscr{D}(a, \alpha(a)]$ which is the full subcategory of $\mathscr{D}$ obtained as the additive closure of all the indecomposable objects with slopes in the interval $(a, \alpha(a)]$, is an abelian category and equivalent to $\operatorname{coh} \mathbb{X}$.

The following lemma is useful. 
Lemma $2.3([5])$. Let $T=\oplus T_{i}$ be an object in $\mathscr{D}$ with indecomposable direct summands $T_{i} \in \mathscr{D}(a, \alpha(a)]$ for some $a \in \mathbb{Q}$. Then $T$ is extension-free in $\mathscr{D}$ if and only if $\mathscr{D}(T, T[1])=0$.

According to [16], the bounded derived category $D^{b}(\operatorname{coh} \mathbb{X})$ of coherent sheaves is triangle equivalent to the stable category $\mathscr{D}$. Thus parallel to [1], we define the cluster category $\mathscr{C}$ to be the orbit category of the stable category $\mathscr{D}$ under the action of the unique auto-equivalence $G=\tau^{-1}[1]$. The cluster category $\mathscr{C}$ has the same objects as $\mathscr{D}$, and for any objects $X, Y$, the homomorphism spaces are given by

$$
\mathscr{C}(X, Y)=\bigoplus_{n \in \mathbb{Z}} \mathscr{D}\left(X, G^{n} Y\right)
$$

with the obvious composition. This orbit category is triangulated and Calabi-Yau of CY-dimension 2, and the canonical functor $\pi: \mathscr{D} \rightarrow \mathscr{C}$ is a triangulated functor[10].

From [11] that, an object $T$ in $\mathscr{C}$ is called a cluster tilting object if

$-\mathscr{C}(T, T[1])=0$.

- $\mathscr{C}(T, X[1])=0$ implies $X \in \operatorname{add}(T)$.

Let $T=\oplus T_{i}$ be an object in $\mathscr{D}$ with each indecomposable direct summand $T_{i} \in \mathscr{D}(a, \alpha(a)]$ for some $a \in \mathbb{Q}$. The following result is from [1] (see also [5]).

Lemma 2.4. The object $T$ is a tilting object in $\mathscr{D}$ if and only if $T$ is a cluster tilting object in $\mathscr{C}$.

The following lemma is useful.

Lemma 2.5 ([3, 9]). Let $\mathcal{C}$ be a Hom-finite 2-CY triangulated category with a cluster tilting object $T$. Let $T_{i}$ be indecomposable and $T=T_{0} \oplus T_{i}$. Then there exists a unique indecomposable $T_{i}^{*}$ non-isomorphic to $T_{i}$ such that $\mathscr{M}_{T_{i}}(T)=T_{0} \oplus T_{i}^{*}$ is cluster tilting. Moreover $T_{i}$ and $T_{i}^{*}$ are linked by the existence of exchange triangles

$$
T_{i} \stackrel{u}{\rightarrow} B \stackrel{v}{\rightarrow} T_{i}^{*} \stackrel{w}{\rightarrow} T_{i}[1] \quad \text { and } \quad T_{i}^{*} \stackrel{u^{\prime}}{\rightarrow} B^{\prime} \stackrel{v^{\prime}}{\rightarrow} T_{i} \stackrel{w^{\prime}}{\longrightarrow} T_{i}^{*}[1],
$$

where $u$ and $u^{\prime}$ are minimal left add $T_{0}$-approximations and $v$ and $v^{\prime}$ are minimal right add $T_{0}$-approximations.

This recursive process of mutations for cluster tilting objects is closely related to the notion of mutations of quivers. Recall that in [6] the mutation of a finite quiver $Q$ without loops and oriented cycles of length 2 (2-cycles for short) at a vertex $i$ is a quiver denoted by $\mathscr{M}_{i}(Q)$ and constructed from $Q$ using the following rule:

(M1) for any couple of arrows $j \rightarrow i \rightarrow k$, add an arrow $j \rightarrow k$;

(M2) reverse the arrows incident with $i$;

(M3) remove a maximal collection of 2-cycles.

Lemma 2.6 ([2]). Let $\mathcal{C}$ be a Hom-finite 2-CY triangulated category with a cluster tilting object $T$. Let $T_{i}$ be an indecomposable direct summand of $T$, and denote by $T^{\prime}$ the cluster tilting object $\mathscr{M}_{T_{i}}(T)$. Denote by $Q_{T}$ (resp. $Q_{T^{\prime}}$ ) the quiver of the endomorphism algebra $\operatorname{End}_{\mathcal{C}}(T)$ (resp. $\left.\operatorname{End}_{\mathcal{C}}\left(T^{\prime}\right)\right)$. Assume that there are no loops and no 2-cycles at the vertex $i$ of $Q_{T}$ (resp. $\left.Q_{T^{\prime}}\right)$ corresponding to the indecomposable $T_{i}$ (resp. $T_{i}^{*}$ ). Then $Q_{T^{\prime}}=\mathscr{M}_{i}\left(Q_{T}\right)$. 


\section{IMPORTANT TRIANGLES IN vect $\mathbb{X}$}

In this section, we will present some triangles, which are crucial for constructing tilting objects in the stable category $\mathscr{D}:=$ vect $\mathbb{X}$ of weight type $\left(p_{1}, p_{2}, p_{3}\right)$. Denote by $\bar{x}_{i}=\vec{x}_{i}+\vec{\omega}$ in $\mathbb{L}$ for $i=1,2,3$.

According to ([12], Corollary 4.14), $\mathscr{D}(E, E(\vec{x})) \neq 0$ if and only if $\vec{x}=0$ or $\bar{x}_{i}$ for $i=1,2,3$. Moreover, $\mathscr{D}\left(E, E\left(\bar{x}_{i}\right)\right) \cong k$ for each $i$. Hence each non-zero morphism $E \rightarrow E\left(\bar{x}_{i}\right)$ fits into a triangle in $\mathscr{D}$. In fact, let $\{i, j, k\}=\{1,2,3\}$, we have

Proposition 3.1. For any $1 \leq i \leq 3$, there exists a triangle in $\mathscr{D}$ :

$$
E \rightarrow E\left(\bar{x}_{i}\right) \rightarrow F_{i} \rightarrow E[1] .
$$

Here, $F_{i}$ depends on the weight type. In more details, $F_{i}$ is given as follows:

(1) if $p_{j}=p_{k}=2$, then $F_{i}=0$;

(2) if $p_{j}=2$ and $p_{k}>2$, then $F_{i}=E\left\langle\left(p_{k}-3\right) \vec{x}_{k}\right\rangle\left(\vec{x}_{k}\right)$;

(3) if $p_{j}, p_{k}>2$, then $F_{i}$ is determined by the non-split exact sequence (for any $t \neq i)$ :

$$
\xi_{i, t}: 0 \rightarrow E\left\langle\left(p_{t}-3\right) \vec{x}_{t}\right\rangle\left(\vec{x}_{t}\right) \rightarrow F_{i} \rightarrow \mathcal{O}\left(\bar{x}_{i}+\bar{x}_{t}\right) \rightarrow 0 ;
$$

moreover, $\operatorname{rk} F_{i}=3$ and

$$
P F_{i}=\left(\bigoplus_{t \neq i}\left(\mathcal{O}\left(\bar{x}_{i}-\vec{x}_{t}\right) \oplus \mathcal{O}\left(\bar{x}_{t}\right)\right)\right) \oplus \mathcal{O}\left(\vec{\omega}+\bar{x}_{i}\right) \oplus \mathcal{O},
$$

and

$$
I F_{i}=\bigoplus_{t=1}^{3}\left(\mathcal{O}\left(\left(p_{t}-1\right) \vec{x}_{t}\right) \oplus \mathcal{O}\left(\bar{x}_{i}+\bar{x}_{t}\right)\right)
$$

Proof From ([12], Corollary 4.14), we have $\mathscr{D}\left(E, E\left(\bar{x}_{i}\right)\right) \neq 0$ for $1 \leq i \leq 3$. Then by [7], any nonzero morphism $f: E \rightarrow E\left(\bar{x}_{i}\right)$ is injective in coh $\mathbb{X}$. Thus we get a short exact sequence

$$
0 \rightarrow E \rightarrow E\left(\bar{x}_{i}\right) \rightarrow \mathcal{S} \rightarrow 0
$$

where $\mathcal{S}$ is a sheaf of finite length.

Notice that by Lemma 4.10 in [12], $\operatorname{det} \mathcal{S}=\operatorname{det} E\left(\bar{x}_{i}\right)-\operatorname{det} E=2 \bar{x}_{i}=\left(p_{j}-\right.$ $2) \vec{x}_{j}+\left(p_{k}-2\right) \vec{x}_{k}$. We have the following three cases to consider:

(1) If $p_{j}=p_{k}=2$, then $\operatorname{det} \mathcal{S}=0$. It follows that $\mathcal{S}=0$, and then $E \cong E\left(\bar{x}_{i}\right)$. Hence $F_{i}=0$.

(2) If $p_{j}=2$ and $p_{k}>2$, then it is easy to verify that $\mathcal{S}=S_{k, p_{k}-2}^{\left(p_{k}-2\right)}$, where $S_{k, p_{k}-2}$ is the unique simple sheaf concentrated at the exceptional point corresponding to $x_{k}$ satisfying $\operatorname{Hom}\left(\mathcal{O}\left(\left(p_{k}-2\right) \vec{x}_{k}\right), S_{k, p_{k}-2}\right) \neq 0$, and $S_{k, p_{k}-2}^{\left(p_{k}-2\right)}$ is the unique torsion sheaf with top $S_{k, p_{k}-2}$ and of length $p_{k}-2$.

Now we make the following pushout commutative diagram:

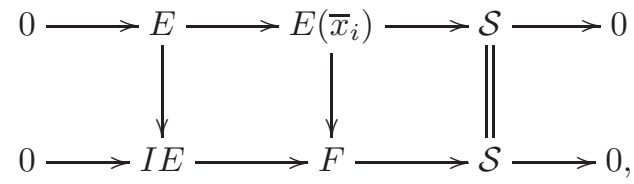

where $I E=\mathcal{O} \oplus\left(\bigoplus_{i=1}^{3} \mathcal{O}\left(\bar{x}_{i}\right)\right)$ is the injective hull of $E$. 
Notice that for any $t \neq k, \operatorname{Ext}^{1}\left(\mathcal{S}, \mathcal{O}\left(\bar{x}_{t}\right)\right)=0$. We obtain that $\mathcal{O}\left(\bar{x}_{t}\right)$ is a direct summand of $F$ for $t \neq k$. By canceling out the common line bundle summands of $I E$ and $F$, we get an exact sequence

$$
0 \rightarrow \mathcal{O} \oplus \mathcal{O}\left(\bar{x}_{k}\right) \rightarrow F_{i} \rightarrow \mathcal{S} \rightarrow 0
$$

Observe that $F_{i}$ is indecomposable of rank two, which is an extension bundle. According to ([12], Theorem 4.2), $F_{i}$ is determined by its class in $K_{0}(\mathbb{X})$. Moreover, $\left[F_{i}\right]=[\mathcal{O}]+\left[\mathcal{O}\left(\bar{x}_{k}\right)\right]+\left[S_{k, p_{k}-2}^{\left(p_{k}-2\right)}\right]=\left[\mathcal{O}\left(\bar{x}_{k}\right)\right]+\left[\mathcal{O}\left(\left(p_{k}-2\right) \vec{x}_{k}\right)\right]=\left[E\left\langle\left(p_{k}-3\right) \vec{x}_{k}\right\rangle\left(\vec{x}_{k}\right)\right]$. Thus we get $F_{i}=E\left\langle\left(p_{k}-3\right) \vec{x}_{k}\right\rangle\left(\vec{x}_{k}\right)$.

(3) If $p_{j}, p_{k}>2$, then $\mathcal{S}=S_{j, p_{j}-2}^{\left(p_{j}-2\right)} \oplus S_{k, p_{k}-2}^{\left(p_{k}-2\right)}$. In the following pushout diagram,

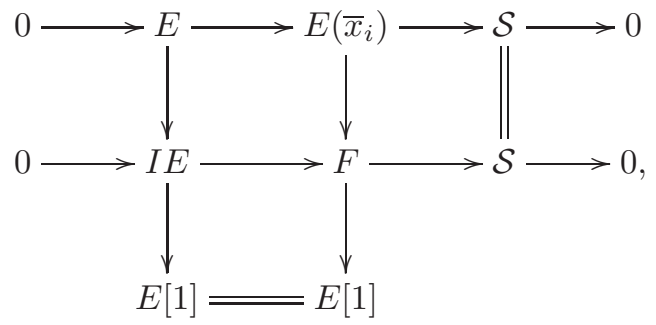

we find that only one of the direct summands, $\mathcal{O}\left(\bar{x}_{i}\right)$, of $I E$ is a direct summand of $F$ since $\operatorname{Ext}^{1}\left(\mathcal{S}, \mathcal{O}\left(\bar{x}_{i}\right)\right)=0$. Hence we get distinguished exact sequences

$$
\zeta_{i}: 0 \rightarrow E \rightarrow E\left(\bar{x}_{i}\right) \oplus I E \rightarrow F \rightarrow 0,
$$

and

$$
\gamma_{i}: 0 \rightarrow E\left(\bar{x}_{i}\right) \rightarrow \mathcal{O}\left(\bar{x}_{i}\right) \oplus F_{i} \rightarrow E[1] \rightarrow 0,
$$

here, $E[1]$ is viewed as an object in vect $\mathbb{X}, F=\mathcal{O}\left(\bar{x}_{i}\right) \oplus F_{i}$ and $F_{i}$ satisfies the following exact sequence

$$
\eta_{i}: 0 \rightarrow \mathcal{O} \oplus \mathcal{O}\left(\bar{x}_{j}\right) \oplus \mathcal{O}\left(\bar{x}_{k}\right) \rightarrow F_{i} \rightarrow \mathcal{S} \rightarrow 0
$$

It follows that

$$
\zeta_{i}^{\prime}: 0 \rightarrow E \rightarrow E\left(\bar{x}_{i}\right) \oplus\left(I E \backslash \mathcal{O}\left(\bar{x}_{i}\right)\right) \rightarrow F_{i} \rightarrow 0
$$

is also a distinguished exact sequence and $F_{i}$ is an indecomposable vector bundle.

Now we claim that $F_{i}$ is determined by the non-split sequence $\xi_{i, t}$. In fact, from the distinguished exact sequence $\gamma_{i}$, we get that the injective hull $I\left(E\left(\bar{x}_{i}\right)\right)$ is a direct summand of $I F_{i} \oplus \mathcal{O}\left(\bar{x}_{i}\right)$. In particular, for any $t \neq i, \mathcal{O}\left(\bar{x}_{i}+\bar{x}_{t}\right)$ is a direct summand of $I F_{i}$. Moreover, applying $\operatorname{Hom}\left(-, \mathcal{O}\left(\bar{x}_{i}+\bar{x}_{t}\right)\right)$ to $\eta_{i}$, we get $\operatorname{Hom}\left(F_{i}, \mathcal{O}\left(\bar{x}_{i}+\bar{x}_{t}\right)\right) \cong k$. Thus, the nonzero morphism $\varphi_{t}: F_{i} \rightarrow \mathcal{O}\left(\bar{x}_{i}+\bar{x}_{t}\right)$ is surjective. We only consider the case of $t=k$, the case of $t=j$ is similar. From the exact sequence

$$
0 \rightarrow \mathcal{O}\left(\bar{x}_{j}\right) \rightarrow \mathcal{O}\left(\bar{x}_{i}+\bar{x}_{k}\right) \rightarrow S_{j, p_{j}-2}^{\left(p_{j}-2\right)} \rightarrow 0
$$

we get $\left[\mathcal{O}\left(\bar{x}_{i}+\bar{x}_{k}\right)\right]=\left[\mathcal{O}\left(\bar{x}_{j}\right)\right]+\left[S_{j, p_{j}-2}^{\left(p_{j}-2\right)}\right]$. It follows from $\eta_{i}$ that $\left[\operatorname{ker} \varphi_{k}\right]=[\mathcal{O}]+$ $\left[\mathcal{O}\left(\bar{x}_{k}\right)\right]+\left[S_{k, p_{k}-2}^{\left(p_{k}-2\right)}\right]=\left[\mathcal{O}\left(\bar{x}_{k}\right)\right]+\left[\mathcal{O}\left(\left(p_{k}-2\right) \vec{x}_{k}\right)\right]=\left[E\left\langle\left(p_{k}-3\right) \vec{x}_{k}\right\rangle\left(\vec{x}_{k}\right)\right]$. Notice that $\operatorname{Ext}^{1}\left(\mathcal{O}\left(\bar{x}_{i}+\bar{x}_{k}\right), E\left\langle\left(p_{k}-3\right) \vec{x}_{k}\right\rangle\left(\vec{x}_{k}\right)\right) \cong k$. Hence, to finish the proof of the claim, we only need to show that $\operatorname{ker} \varphi_{k}$ is indecomposable. In fact, if $\operatorname{ker} \varphi_{k}=\mathcal{O}\left(\vec{y}_{1}\right) \oplus \mathcal{O}\left(\vec{y}_{2}\right)$, then applying $\operatorname{Hom}\left(\mathcal{O}\left(\bar{x}_{k}\right),-\right)$ to the exact sequence

$$
\theta: 0 \rightarrow \mathcal{O}\left(\vec{y}_{1}\right) \oplus \mathcal{O}\left(\vec{y}_{2}\right) \rightarrow F_{i} \rightarrow \mathcal{O}\left(\bar{x}_{i}+\bar{x}_{k}\right) \rightarrow 0,
$$


we get $\operatorname{Hom}\left(\mathcal{O}\left(\bar{x}_{k}\right), \mathcal{O}\left(\vec{y}_{l}\right)\right) \neq 0$ for $l=1$ or 2 , thus $\vec{y}_{l}-\bar{x}_{k} \geq 0$. On the other hand, applying $\operatorname{Ext}^{1}\left(-, \mathcal{O}\left(\vec{y}_{l}\right)\right)$ to $\theta$, we get $\operatorname{Ext}^{1}\left(\mathcal{O}\left(\bar{x}_{i}+\bar{x}_{k}\right), \mathcal{O}\left(\vec{y}_{l}\right)\right) \cong D \operatorname{Hom}\left(\mathcal{O}\left(\vec{y}_{l}\right), \mathcal{O}\left(\bar{x}_{i}+\right.\right.$ $\left.\left.\bar{x}_{k}+\vec{\omega}\right)\right) \neq 0$. It follows that $\bar{x}_{i}+\bar{x}_{k}+\vec{\omega}-\vec{y}_{l} \geq 0$. Hence, $\vec{y}_{l}-\bar{x}_{k} \leq \bar{x}_{i}+\vec{\omega}=$ $\vec{\omega}+\vec{c}-\vec{x}_{j}-\vec{x}_{k}<\vec{\omega}+\vec{c}$, a contradiction. The claim is proved.

Moreover, from the distinguished exact sequence $\zeta_{i}$, we get that the projective cover $P F$ of $F$ is a direct summand of $P\left(E\left(\bar{x}_{i}\right) \oplus I E\right)=P\left(E\left(\bar{x}_{i}\right)\right) \oplus I E$, where $P\left(E\left(\bar{x}_{i}\right)\right)=\mathcal{O}\left(\bar{x}_{i}+\vec{\omega}\right) \oplus \mathcal{O}\left(\bar{x}_{i}-\vec{x}_{i}\right) \oplus \mathcal{O}\left(\bar{x}_{i}-\vec{x}_{j}\right) \oplus \mathcal{O}\left(\bar{x}_{i}-\vec{x}_{k}\right)$. From the fact that the shift functor preserves the rank, we get $\operatorname{rk}\left(P F_{i}\right)=2 \operatorname{rk} F_{i}=6$. Notice that $\operatorname{Hom}\left(\mathcal{O}\left(\bar{x}_{i}-\vec{x}_{i}\right), F_{i}\right) \cong k$, and each morphism $f: \mathcal{O}\left(\bar{x}_{i}-\vec{x}_{i}\right) \rightarrow F_{i}$ factors through the direct summand $\mathcal{O}\left(\bar{x}_{j}\right)$ of $I E$. Hence, $\mathcal{O}\left(\bar{x}_{i}-\vec{x}_{i}\right)$ is not a summand of $P F_{i}$. Thus, $P F_{i}=P\left(E\left(\bar{x}_{i}\right) \oplus I E\right) \backslash\left(\mathcal{O}\left(\bar{x}_{i}\right) \oplus \mathcal{O}\left(\bar{x}_{i}-\vec{x}_{i}\right)\right)=\mathcal{O}\left(\bar{x}_{i}+\vec{\omega}\right) \oplus \mathcal{O}\left(\bar{x}_{i}-\vec{x}_{j}\right) \oplus$ $\mathcal{O}\left(\bar{x}_{i}-\vec{x}_{k}\right) \oplus \mathcal{O}\left(\bar{x}_{j}\right) \oplus \mathcal{O}\left(\bar{x}_{k}\right) \oplus \mathcal{O}$.

In order to calculate the injective hull $I F_{i}$ of $F_{i}$, we consider the following pushout diagram

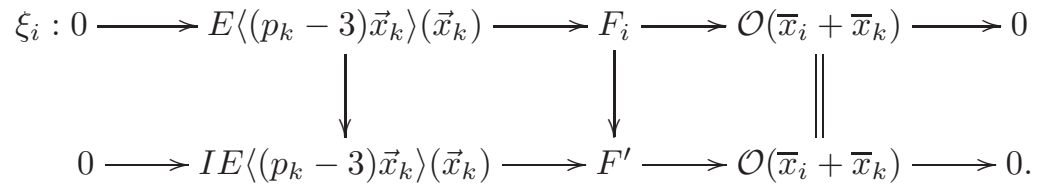

Recall that $I E\left\langle\left(p_{k}-3\right) \vec{x}_{k}\right\rangle\left(\vec{x}_{k}\right)=\mathcal{O}\left(\left(p_{k}-2\right) \vec{x}_{k}\right) \oplus \mathcal{O}\left(\vec{x}_{k}+\bar{x}_{i}\right) \oplus \mathcal{O}\left(\vec{x}_{k}+\bar{x}_{j}\right) \oplus \mathcal{O}(\vec{\omega}+$ $\left.\left(p_{k}-1\right) \vec{x}_{k}\right)$. It is easy to verify that $\operatorname{Ext}^{1}\left(\mathcal{O}\left(\bar{x}_{i}+\bar{x}_{k}\right), L\right)=0$ for any direct summand $L$ of $I\left(E\left\langle\left(p_{k}-3\right) \vec{x}_{k}\right\rangle\left(\vec{x}_{k}\right)\right)$ different from $\mathcal{O}\left(\left(p_{k}-2\right) \vec{x}_{k}\right)$, that is, $\mathcal{O}\left(\vec{x}_{k}+\bar{x}_{i}\right) \oplus \mathcal{O}\left(\vec{x}_{k}+\right.$ $\left.\bar{x}_{j}\right) \oplus \mathcal{O}\left(\vec{\omega}+\left(p_{k}-1\right) \vec{x}_{k}\right)$ is a direct summand of $F^{\prime}$. Moreover, the middle term of the non-split exact sequence

$$
0 \rightarrow \mathcal{O}\left(\left(p_{k}-2\right) \vec{x}_{k}\right) \rightarrow E^{\prime} \rightarrow \mathcal{O}\left(\bar{x}_{i}+\bar{x}_{k}\right) \rightarrow 0
$$

has the expression $E^{\prime}=E\left\langle\vec{x}_{j}\right\rangle\left(\bar{x}_{i}\right)$ by considering its class in $K_{0}(\mathbb{X})$ : $\left[E^{\prime}\right]=\left[\mathcal{O}\left(\left(p_{k}-\right.\right.\right.$ $\left.\left.2) \vec{x}_{k}\right)\right]+\left[\mathcal{O}\left(\bar{x}_{i}+\bar{x}_{k}\right)\right]=\left[\mathcal{O}\left(\left(p_{k}-1\right) \vec{x}_{k}\right)\right]+\left[\mathcal{O}\left(\bar{x}_{i}+\vec{\omega}\right)\right]=\left[E\left\langle\vec{x}_{j}\right\rangle\left(\bar{x}_{i}\right)\right]$. Thus $F^{\prime}=$ $\mathcal{O}\left(\vec{x}_{k}+\bar{x}_{i}\right) \oplus \mathcal{O}\left(\vec{x}_{k}+\bar{x}_{j}\right) \oplus \mathcal{O}\left(\vec{\omega}+\left(p_{k}-1\right) \vec{x}_{k}\right) \oplus E\left\langle\vec{x}_{j}\right\rangle\left(\bar{x}_{i}\right)$. Since distinguished injectivity is preserved under taking pushout, we have $F_{i} \hookrightarrow F^{\prime}$ is a distinguished injection, which implies that $I F_{i}$ is a direct summand of $I F^{\prime}$. Recall that $I\left(E\left\langle\vec{x}_{j}\right\rangle\left(\bar{x}_{i}\right)\right)=$ $\mathcal{O}\left(\bar{x}_{i}+\vec{x}_{j}\right) \oplus \mathcal{O}\left(2 \bar{x}_{i}\right) \oplus \mathcal{O}\left(\bar{x}_{i}+\bar{x}_{k}\right) \oplus \mathcal{O}\left(\bar{x}_{i}+\bar{x}_{j}+\vec{x}_{j}\right)$. It is easy to see that $\operatorname{Hom}(\mathcal{O}(\vec{\omega}+$ $\left.\left.\left(p_{k}-1\right) \vec{x}_{k}\right), \mathcal{O}\left(\bar{x}_{i}+\bar{x}_{j}+\vec{x}_{j}\right)\right) \cong S_{\vec{x}_{j}} \cong k$ and $\operatorname{Hom}\left(F_{i}, \mathcal{O}\left(\bar{x}_{i}+\bar{x}_{j}+\vec{x}_{j}\right)\right) \cong k$. Thus each morphism $f: F_{i} \rightarrow \mathcal{O}\left(\bar{x}_{i}+\bar{x}_{j}+\vec{x}_{j}\right)$ factors through $\mathcal{O}\left(\vec{\omega}+\left(p_{k}-1\right) \vec{x}_{k}\right)$. We conclude that $\mathcal{O}\left(\bar{x}_{i}+\bar{x}_{j}+\vec{x}_{j}\right)$ is not a summand of $I F_{i}$. Thus, in view of the rank, we have $I F_{i}=\bigoplus_{t=1}^{3}\left(\mathcal{O}\left(\left(p_{t}-1\right) \vec{x}_{t}\right) \oplus \mathcal{O}\left(\bar{x}_{i}+\bar{x}_{t}\right)\right)$.

Proposition 3.2. Assume $0 \leq \vec{x}-\vec{x}_{j}-\vec{x}_{k} \leq \vec{x} \leq 2 \vec{\omega}+\vec{c}$. Then there is a triangle in $\mathscr{D}$ :

$$
\xi: G[-1] \rightarrow E\left\langle\vec{x}-\vec{x}_{j}\right\rangle \oplus E\left\langle\vec{x}-\vec{x}_{k}\right\rangle \rightarrow E\langle\vec{x}\rangle \rightarrow G,
$$

where $G$ is determined by the following non-split exact sequence in coh $\mathbb{X}$ :

$$
\zeta: 0 \rightarrow E\left\langle\vec{x}-\vec{x}_{j}-\vec{x}_{k}\right\rangle[1] \rightarrow G \rightarrow \mathcal{O}(\vec{x}) \rightarrow 0 .
$$

Proof Write $\vec{x}=\sum_{i=1}^{3} l_{i} \vec{x}_{i}$, by Proposition 4.20 in [12] there is a triangle in $\mathscr{D}$

$$
\eta: E\left\langle\vec{x}-\vec{x}_{j}\right\rangle \rightarrow E\langle\vec{x}\rangle \rightarrow E\left\langle\vec{x}-l_{j} \vec{x}_{j}\right\rangle\left(l_{j} \vec{x}_{j}\right) \rightarrow E\left\langle\vec{x}-\vec{x}_{j}\right\rangle[1] .
$$


Then $\eta$ induces the following homotopy pullback commutative diagram([8])

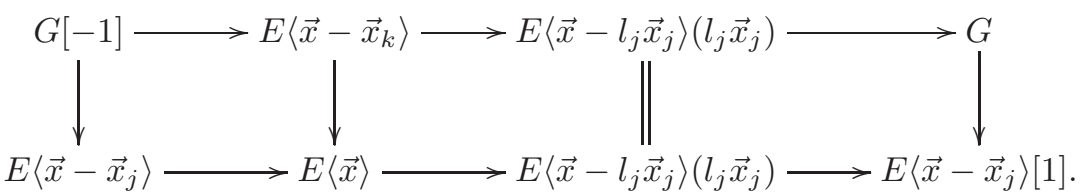

It follows that

$$
\xi: G[-1] \rightarrow E\left\langle\vec{x}-\vec{x}_{j}\right\rangle \oplus E\left\langle\vec{x}-\vec{x}_{k}\right\rangle \rightarrow E\langle\vec{x}\rangle \rightarrow G
$$

is a triangle in $\mathscr{D}$.

Now we claim that $G$ is determined by the exact sequence $\zeta$. In fact, there is an exact sequence in $\operatorname{coh} \mathbb{X}$

$$
\theta: 0 \rightarrow E\left\langle\vec{x}-\vec{x}_{k}\right\rangle \rightarrow E\left\langle\vec{x}-l_{j} \vec{x}_{j}\right\rangle\left(l_{j} \vec{x}_{j}\right) \rightarrow \mathcal{S} \rightarrow 0,
$$

where by calculating the determinant $\operatorname{det} \mathcal{S}=l_{j} \vec{x}_{j}+\vec{x}_{k}$. We conclude that $\mathcal{S}=$ $S_{j, l_{j}-1}^{\left(l_{j}\right)} \oplus S_{k, l_{k}}$. Notice that $\theta$ induces the pushout commutative diagram

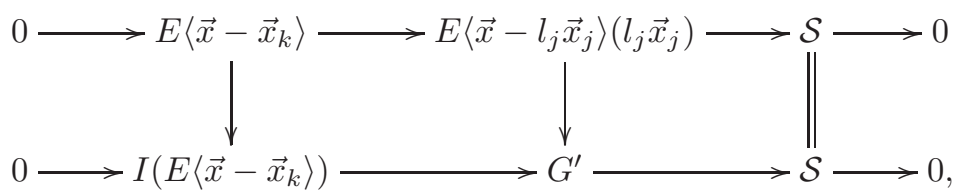

where $I\left(E\left\langle\vec{x}-\vec{x}_{k}\right\rangle\right)=\mathcal{O}\left(\vec{x}-\vec{x}_{k}\right) \oplus \mathcal{O}\left(\vec{\omega}+\left(l_{i}+1\right) \vec{x}_{i}\right) \oplus \mathcal{O}\left(\vec{\omega}+\left(l_{j}+1\right) \vec{x}_{j}\right) \oplus \mathcal{O}\left(\vec{\omega}+l_{k} \vec{x}_{k}\right)$ is the injective hull of $E\left\langle\vec{x}-\vec{x}_{k}\right\rangle$. It is easy to find that only one direct summand, $\mathcal{O}\left(\vec{\omega}+\left(l_{j}+1\right) \vec{x}_{j}\right)$, of $I\left(E\left\langle\vec{x}-\vec{x}_{k}\right\rangle\right)$ vanishes under the functor $\operatorname{Ext}^{1}(\mathcal{S},-)$. Thus $\mathcal{O}\left(\vec{\omega}+\left(l_{j}+1\right) \vec{x}_{j}\right)$ is a direct summand of $G^{\prime}$. By using similar arguments as shown in Proposition 3.1, we get that $G^{\prime}=\mathcal{O}\left(\vec{\omega}+\left(l_{j}+1\right) \vec{x}_{j}\right) \oplus G$ for an indecomposable sheaf $G$. From the distinguished injection $E\left\langle\vec{x}-l_{j} \vec{x}_{j}\right\rangle\left(l_{j} \vec{x}_{j}\right) \hookrightarrow G^{\prime}$, we get that the injective hull of $E\left\langle\vec{x}-l_{j} \vec{x}_{j}\right\rangle\left(l_{j} \vec{x}_{j}\right)$ is a direct summand of $I G^{\prime}$. In particular, $\mathcal{O}(\vec{x})$ is a direct summand of $I G$, which induces an epimorphism $\varphi: G \rightarrow \mathcal{O}(\vec{x})$. We claim that $\operatorname{ker} \varphi$ is indecomposable. Otherwise, $\operatorname{ker} \varphi=\mathcal{O}\left(\vec{y}_{1}\right) \oplus \mathcal{O}\left(\vec{y}_{2}\right)$ for some $\vec{y}_{1}, \vec{y}_{2} \in \mathbb{L}$. Applying $\operatorname{Hom}\left(\mathcal{O}\left(\vec{\omega}+\left(l_{i}+1\right) \vec{x}_{i}\right),-\right)$ to the exact sequence

$$
\theta^{\prime}: 0 \rightarrow \mathcal{O}\left(\vec{y}_{1}\right) \oplus \mathcal{O}\left(\vec{y}_{2}\right) \rightarrow G \rightarrow \mathcal{O}(\vec{x}) \rightarrow 0
$$

we get $\operatorname{Hom}\left(\mathcal{O}\left(\vec{\omega}+\left(l_{i}+1\right) \vec{x}_{i}\right), \mathcal{O}\left(\vec{y}_{t}\right)\right) \neq 0$ for some $t=1,2$. Hence, $\vec{y}_{t} \geq \vec{\omega}+\left(l_{i}+1\right) \vec{x}_{i}$. On the other hand, applying $\operatorname{Ext}^{1}\left(-, \mathcal{O}\left(\vec{y}_{t}\right)\right)$ to $\theta^{\prime}$, we get $\operatorname{Ext}^{1}\left(\mathcal{O}(\vec{x}), \mathcal{O}\left(\vec{y}_{t}\right)\right)=$ $D \operatorname{Hom}\left(\mathcal{O}\left(\vec{y}_{t}\right), \mathcal{O}(\vec{x}+\vec{\omega})\right) \neq 0$. Thus $\vec{\omega}+\left(l_{i}+1\right) \vec{x}_{i} \leq \vec{y}_{t} \leq \vec{x}+\vec{\omega}$, a contradiction, as claimed. Therefore, $\operatorname{ker} \varphi$ is an extension bundle, which is determined by its class in $K_{0}(\mathbb{X})$. Notice that $[\operatorname{ker} \varphi]=[G]-[\mathcal{O}(\vec{x})]=\left[\mathcal{O}\left(\vec{\omega}+\left(l_{i}+1\right) \vec{x}_{i}\right)\right]+\left[\mathcal{O}\left(\vec{\omega}+l_{k} \vec{x}_{k}\right)\right]+$ $\left[S_{j, l_{j}-1}^{\left(l_{j}\right)}\right]=\left[\mathcal{O}\left(\vec{\omega}+\left(l_{i}+1\right) \vec{x}_{i}+l_{j} \vec{x}_{j}\right)\right]+\left[\mathcal{O}\left(\vec{\omega}+l_{k} \vec{x}_{k}\right)\right]=\left[E\left\langle l_{i} \vec{x}_{i}+\left(l_{j}-1\right) \vec{x}_{j}+\left(p_{k}-\right.\right.\right.$ $\left.\left.\left.1-l_{k}\right) \vec{x}_{k}\right\rangle\left(l_{k} \vec{x}_{k}\right)\right]=\left[E\left\langle\vec{x}-\vec{x}_{j}-\vec{x}_{k}\right\rangle[1]\right]$. We obtain the exact sequence $\xi$. Moreover, the fact that $\operatorname{Ext}^{1}\left(\mathcal{O}(\vec{x}), E\left\langle l_{i} \vec{x}_{i}+\left(l_{j}-1\right) \vec{x}_{j}+\left(p_{k}-1-l_{k}\right) \vec{x}_{k}\right\rangle\left(l_{k} \vec{x}_{k}\right)\right) \cong k$ implies that the middle term $G$ of $\xi$ is uniquely determined.

By using the triangle

$$
E\langle\vec{x}\rangle \rightarrow E\left\langle\vec{x}+\vec{x}_{j}\right\rangle \rightarrow E\left\langle\vec{x}-l_{j} \vec{x}_{j}\right\rangle\left(\left(l_{j}+1\right) \vec{x}_{j}\right) \rightarrow E\langle\vec{x}\rangle[1]
$$


and the induced homotopy pushout commutative diagram

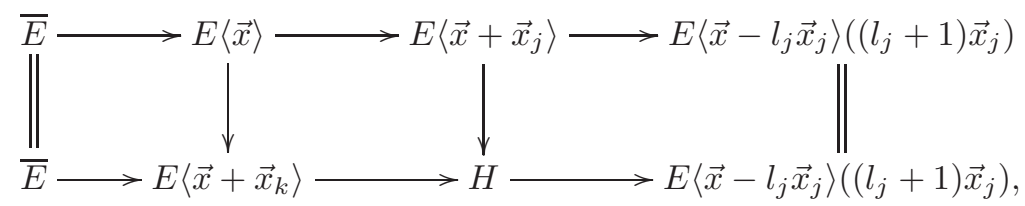

where $\bar{E}=E\left\langle\vec{x}+\left(p_{j}-2-l_{j}\right) \vec{x}_{j}\right\rangle\left(\left(l_{j}+2-p_{j}\right) \vec{x}_{j}\right)$, we have the following similar result.

Proposition 3.3. Assume $0 \leq \vec{x} \leq \vec{x}+\vec{x}_{j}+\vec{x}_{k} \leq 2 \vec{\omega}+\vec{c}$. Then there is a triangle in $\mathscr{D}$ :

$$
H[-1] \rightarrow E\langle\vec{x}\rangle \rightarrow E\left\langle\vec{x}+\vec{x}_{j}\right\rangle \oplus E\left\langle\vec{x}+\vec{x}_{k}\right\rangle \rightarrow H,
$$

where $H$ is determined by the following non-split exact sequence:

$$
0 \rightarrow E\left\langle\vec{x}+\vec{x}_{j}\right\rangle \rightarrow H \rightarrow \mathcal{O}\left(\vec{x}+\vec{x}_{k}\right) \rightarrow 0 .
$$

\section{Tubular tilting OBJECTS IN vect $\mathbb{X}$}

We focus on the tubular tilting objects in the stable category of vector bun-

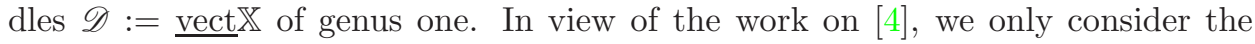
weighted projective line of genus one and weight triple, that is, $\mathbb{X}$ is of weight type $(2,4,4),(2,3,6)$ or $(3,3,3)$. We start with studying the properties of tilting objects in $\mathscr{D}$.

Lemma 4.1. Assume that $E \oplus F$ is extension-free in $\mathscr{D}$ and $\mu F=\mu(E[1])$. Then $F \oplus\left(\tau^{-1} E[1]\right)$ is extension-free if and only if $\mathscr{D}(F, E(\vec{c}-\vec{\omega}))=0$.

Proof Notice that $\mu F=\mu(E[1])$, by ([12], Theorem A.6) and the semi-stability of vector bundles, we have $\mathscr{D}\left(\tau^{-1} E[1], F[n]\right)=0=\mathscr{D}\left(F,\left(\tau^{-1} E[1]\right)[n]\right)$ for any $n \neq 0,1$. Moreover, the assumption that $E \oplus F$ is extension-free implies that $\mathscr{D}\left(\tau^{-1} E[1], F[1]\right) \cong D \mathscr{D}(F, E[1])=0$. Thus, the result is easily obtained since $\mathscr{D}\left(F,\left(\tau^{-1} E[1]\right)[1]\right)=\mathscr{D}(F, E(\vec{c}-\vec{\omega}))$.

Proposition 4.2. Let $T=\bigoplus_{i \in I} T_{i}$ be a tilting object in $\mathscr{D}$ with indecomposable direct summand $T_{i} \in \mathscr{D}[a, \alpha(a)]$ for some $a \in \mathbb{Q}$. Assume that $I_{1}=\left\{i \in I \mid \mu T_{i}=a\right\}$ and $I_{2}=\left\{i \in I \mid \mu T_{i}=\alpha(a)\right\}$. Then $T^{\prime}=\left(\bigoplus_{i \in I_{1}} \tau^{-1} T_{i}[1]\right) \oplus\left(\bigoplus_{j \in I \backslash I_{1}} T_{j}\right)$ is a tilting object in $\mathscr{D}$ if and only if $\mathscr{D}\left(T_{i_{2}}, T_{i_{1}}(\vec{c}-\vec{\omega})\right)=0$ for any $i_{1} \in I_{1}$ and $i_{2} \in I_{2}$.

Proof Notice that each direct summand of $T^{\prime}$ belongs to $\mathscr{D}(a, \alpha(a)]$. Hence by ([12], Theorem A.6) and the semi-stability of vector bundles, for any $i \in$ $I_{1}$ and $t \in I \backslash\left(I_{1} \cup I_{2}\right)$, we have $\mathscr{D}\left(T_{t},\left(\tau^{-1} T_{i}[1]\right)[n]\right)=0$ for any $n \neq 0$ and $\mathscr{D}\left(\tau^{-1} T_{i}[1], T_{t}[n]\right)=0$ for any $n \neq 1$. Moreover, if $n=1$, then by Serre duality, we have $\mathscr{D}\left(\tau^{-1} T_{i}[1], T_{t}[1]\right)=D \mathscr{D}\left(T_{t}, T_{i}[1]\right)=0$. Thus, $T^{\prime}$ is extension-free if and only if $\tau^{-1} T_{i_{1}}[1] \oplus T_{i_{2}}$ is extension-free for any $i_{1} \in I_{1}$ and $i_{2} \in I_{2}$, that is, $\mathscr{D}\left(T_{i_{2}}, T_{i_{1}}(\vec{c}-\vec{\omega})\right)=0$ by the preceding lemma. Notice that the direct summand of $T^{\prime}$ can be arranged as a complete exceptional sequence, thus $T^{\prime}$ is automatically a tilting object.

Since $T_{\text {cub }}$ is a tilting object in $\mathscr{D}$, the direct summands of $T_{\text {cub }}$ belong to $\mathscr{D}[0, \alpha(0)]$, and only one of them has minimal (respectively, maximal) slope, that 
is, $\mu E=0$ and $\mu(E\langle 2 \vec{\omega}+\vec{c}\rangle)=\frac{\delta(\vec{c})}{2}=\alpha(0)$. Then by Proposition 4.2, we have the following results.

Corollary 4.3. Let $T^{\prime}=\tau^{-1} E[1] \oplus(\underset{0<\vec{x} \leq 2 \vec{\omega}+\vec{c}}{\bigoplus} E\langle\vec{x}\rangle)$.

(1) If weight type of $\mathbb{X}$ is $(2,3,6)$ or $(2,4,4)$, then $T^{\prime}$ is a tilting object in $\mathscr{D}$.

(2) If weight type of $\mathbb{X}$ is $(3,3,3)$, then $T^{\prime}$ is not a tilting object in $\mathscr{D}$.

Proof (1) If $\mathbb{X}$ has weight type $(2,3,6)$ or $(2,4,4)$, then by ([12], Proposition $4.15), E\langle 2 \vec{\omega}+\vec{c}\rangle=E\left(\left(p_{2}-1\right) \vec{x}_{2}+\left(p_{3}-1\right) \vec{x}_{3}-\vec{c}\right)$. Thus $\mathscr{D}(E\langle 2 \vec{\omega}+\vec{c}\rangle, E(\vec{c}-\vec{\omega}))=$ $\mathscr{D}\left(E, E\left(\vec{x}_{1}+2 \vec{x}_{2}+2 \vec{x}_{3}-\vec{c}\right)\right)=0$ since $\vec{x}_{1}+2 \vec{x}_{2}+2 \vec{x}_{3}-\vec{c} \neq 0$ or $\bar{x}_{i}$ for $1 \leq i \leq 3$. Then by Proposition 4.2, $T^{\prime}$ is a tilting object in $\mathscr{D}$.

(2) If $\mathbb{X}$ has weight type $(3,3,3)$, then by ([12], Proposition 4.15), $E\langle 2 \vec{\omega}+\vec{c}\rangle=$ $E\left\langle\vec{x}_{1}+\vec{x}_{2}+\vec{x}_{3}\right\rangle=(E[1])(\vec{\omega})$. Thus $\mathscr{D}(E\langle 2 \vec{\omega}+\vec{c}\rangle, E(\vec{c}-\vec{\omega}))=\mathscr{D}(\tau E[1], E(\vec{c}-\vec{\omega}))=$ $\mathscr{D}(\tau E[2], E(\vec{c}-\vec{\omega})[1])=D \mathscr{D}(E(\vec{c}-\vec{\omega}), E(2 \vec{\omega}+\vec{c}))=D \mathscr{D}(E, E) \neq 0$. By Proposition $4.2, T^{\prime}$ is not a tilting object in $\mathscr{D}$.

Now we begin to construct a tubular tilting object in $\mathscr{D}$ case by case. Firstly, we consider $\mathbb{X}$ of weight type $(2,4,4)$. The main strategy is to use the cluster mutation. More precisely, we first construct an original cluster tilting object in $\mathscr{C}$ from $T_{\text {cub }}$, then by a sequence of tubular mutations based on Keller's soft-ware we deduce a tilting object in $\mathscr{D}$ with tubular endomorphism algebra.

Theorem 4.4. Assume that $\mathbb{X}$ has weight type $(2,4,4)$. Then there exists a tilting object in $\mathscr{D}: T_{(2,4,4)}=\tau^{-1}\left(E^{*} \oplus E\left\langle\vec{x}_{2}\right\rangle \oplus E\left\langle\vec{x}_{3}\right\rangle \oplus E(-\vec{\omega})\right)[1] \oplus\left(E\left\langle 2 \vec{x}_{2}+\vec{x}_{3}\right\rangle \oplus E\left\langle\vec{x}_{2}+\right.\right.$ $\left.\left.2 \vec{x}_{3}\right\rangle \oplus E\left(\vec{x}_{1}-\vec{x}_{2}+\vec{x}_{3}\right) \oplus E\left(\vec{x}_{1}+\vec{x}_{2}-\vec{x}_{3}\right) \oplus E\left\langle 2 \vec{x}_{2}+2 \vec{x}_{3}\right\rangle^{*}\right)$, whose endomorphism algebra is a tubular algebra of type $(2,4,4)$, where $E^{*}$ and $E\left\langle 2 \vec{x}_{2}+2 \vec{x}_{3}\right\rangle^{*}$ are determined by the following exact sequences:

$$
0 \rightarrow E\left\langle\vec{x}_{3}\right\rangle \rightarrow E^{*} \rightarrow \mathcal{O}\left(\vec{x}_{2}\right) \rightarrow 0
$$

and

$$
0 \rightarrow E\left\langle\vec{x}_{2}+\vec{x}_{3}\right\rangle \rightarrow E\left\langle 2 \vec{x}_{2}+2 \vec{x}_{3}\right\rangle^{*} \rightarrow \mathcal{O}\left(2 \vec{x}_{2}+2 \vec{x}_{3}-\vec{x}_{1}\right) \rightarrow 0
$$

Proof By Corollary 4.3, we get that $T^{\prime}=\left(\tau^{-1} E[1]\right) \oplus\left(\underset{0<\vec{x} \leq 2 \vec{\omega}+\vec{c}}{\bigoplus_{c}} E\langle\vec{x}\rangle\right)$ is a tilting object in $\mathscr{D}$, and each direct summand of $T^{\prime}$ belongs to $\mathscr{D}(0, \alpha(0)]$. Hence by Lemma $2.4, T^{\prime}$ is a cluster tilting object in $\mathscr{C}$. Notice that $T^{\prime}=T_{\text {cub }}$ in $\mathscr{C}$, the Gabriel quiver of the endomorphism algebra $\mathscr{C}\left(T^{\prime}, T^{\prime}\right)$ has the form:

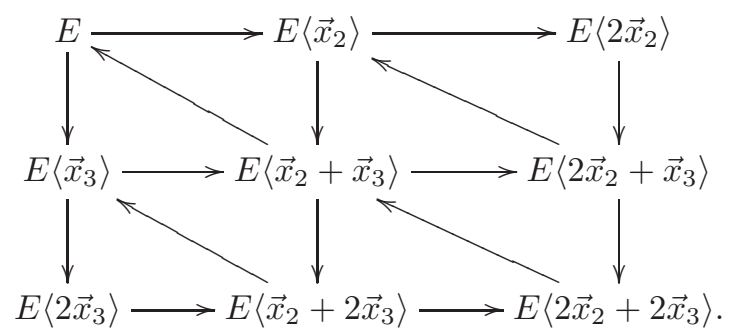


Using Keller's soft-ware, we know that under taking quiver mutations $u_{1} u_{2} u_{3} u_{4} u_{5}$ for the quiver

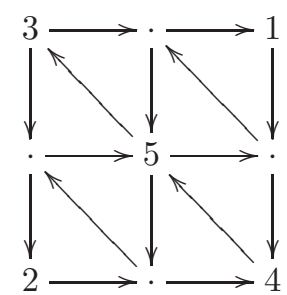

we can obtain the following quiver

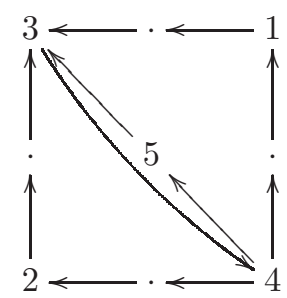

By Lemma 2.6, the quiver mutation $u_{i}$ corresponds to the cluster tilting mutation in the triangulated category $\mathscr{C}$ at the corresponding indecomposable direct summand of $T^{\prime}$. In more details, the cluster tilting mutation corresponding to $u_{i}$ for $1 \leq i \leq 5$ is given as follows.

(1) $u_{1}$ corresponds to the following triangle

$$
E\left\langle 2 \vec{x}_{2}\right\rangle \rightarrow E\left\langle 2 \vec{x}_{2}+\vec{x}_{3}\right\rangle \rightarrow E\left\langle 2 \vec{x}_{2}\right\rangle^{*} \rightarrow E\left\langle 2 \vec{x}_{2}\right\rangle[1],
$$

where $E\left\langle 2 \vec{x}_{2}\right\rangle^{*}=E\left\langle 2 \vec{x}_{2}\right\rangle\left(\vec{x}_{3}\right)=E\left(\vec{x}_{1}-\vec{x}_{2}+\vec{x}_{3}\right)$ by Lemma 2.1;

(2) $u_{2}$ corresponds to the following triangle

$$
E\left\langle 2 \vec{x}_{3}\right\rangle \rightarrow E\left\langle\vec{x}_{2}+2 \vec{x}_{3}\right\rangle \rightarrow E\left\langle 2 \vec{x}_{3}\right\rangle^{*} \rightarrow E\left\langle 2 \vec{x}_{3}\right\rangle[1],
$$

where $E\left\langle 2 \vec{x}_{3}\right\rangle^{*}=E\left\langle 2 \vec{x}_{3}\right\rangle\left(\vec{x}_{2}\right)=E\left(\vec{x}_{1}+\vec{x}_{2}-\vec{x}_{3}\right)$ by Lemma 2.1 ;

(3) $u_{3}$ corresponds to the following triangle

$$
E \rightarrow E\left\langle\vec{x}_{2}\right\rangle \oplus E\left\langle\vec{x}_{3}\right\rangle \rightarrow E^{*} \rightarrow E[1],
$$

where $E^{*}$ is determined by the non-split exact sequence

$$
0 \rightarrow E\left\langle\vec{x}_{3}\right\rangle \rightarrow E^{*} \rightarrow \mathcal{O}\left(\vec{x}_{2}\right) \rightarrow 0 ;
$$

(4) $u_{4}$ corresponds to the following triangle

$$
E\left\langle 2 \vec{x}_{2}+2 \vec{x}_{3}\right\rangle^{*} \rightarrow E\left\langle\vec{x}_{2}+2 \vec{x}_{3}\right\rangle \oplus E\left\langle 2 \vec{x}_{2}+\vec{x}_{3}\right\rangle \rightarrow E\left\langle 2 \vec{x}_{2}+2 \vec{x}_{3}\right\rangle,
$$

where $E\left\langle 2 \vec{x}_{2}+2 \vec{x}_{3}\right\rangle^{*}$ is determined by the non-split exact sequence

$$
0 \rightarrow E\left\langle\vec{x}_{2}+\vec{x}_{3}\right\rangle \rightarrow E\left\langle 2 \vec{x}_{2}+2 \vec{x}_{3}\right\rangle^{*} \rightarrow \mathcal{O}\left(2 \vec{x}_{2}+2 \vec{x}_{3}-\vec{x}_{1}\right) \rightarrow 0 ;
$$

(5) $u_{5}$ corresponds to the following triangle

$$
E\left\langle\vec{x}_{2}+\vec{x}_{3}\right\rangle[-1] \rightarrow E\left\langle\vec{x}_{2}+\vec{x}_{3}\right\rangle^{*} \rightarrow E^{*} \stackrel{\varphi}{\rightarrow} E\left\langle\vec{x}_{2}+\vec{x}_{3}\right\rangle .
$$

Now we claim that $E\left\langle\vec{x}_{2}+\vec{x}_{3}\right\rangle^{*}=E(-\vec{\omega})$. In fact, from (4.1), we have $\mu\left(E^{*}\right)=\frac{2}{3}$, then by comparing the slopes of $E^{*}$ and $E\left\langle\vec{x}_{2}+\vec{x}_{3}\right\rangle$, we obtain that the nonzero morphism $\varphi$ in $\operatorname{coh} \mathbb{X}$ is surjective. Moreover, $\operatorname{det}(\operatorname{ker} \varphi)=\operatorname{det}\left(E^{*}\right)-\operatorname{det}\left(E\left\langle\vec{x}_{2}+\right.\right.$ $\left.\left.\vec{x}_{3}\right\rangle\right)=0$ implies that $\operatorname{ker} \varphi=0$. Thus we get an exact sequence

$$
0 \rightarrow \mathcal{O} \rightarrow E^{*} \stackrel{\varphi}{\rightarrow} E\left\langle\vec{x}_{2}+\vec{x}_{3}\right\rangle \rightarrow 0 .
$$


Taking pullback along (4.3), we get the following commutative diagram

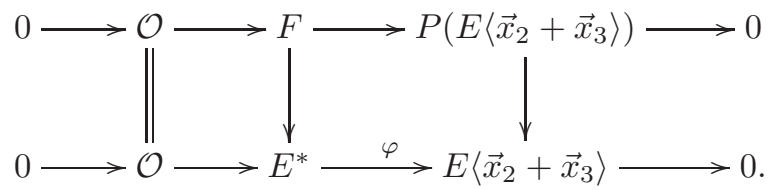

Recall that $P\left(E\left\langle\vec{x}_{2}+\vec{x}_{3}\right\rangle\right)=\mathcal{O}(\vec{\omega}) \oplus \mathcal{O}\left(\vec{x}_{2}-\vec{x}_{3}\right) \oplus \mathcal{O}\left(\vec{x}_{3}-\vec{x}_{2}\right) \oplus \mathcal{O}(-\vec{\omega})$. It is easy to check that all the indecomposable summands but $\mathcal{O}(-\vec{\omega})$ of $P\left(E\left\langle\vec{x}_{2}+\vec{x}_{3}\right\rangle\right)$ vanish under the functor $\operatorname{Ext}^{1}(-, \mathcal{O})$. That is, the other three direct summands of $P\left(E\left\langle\vec{x}_{2}+\vec{x}_{3}\right\rangle\right)$ are direct summands of $F$. It follows that $F=\mathcal{O}(\vec{\omega}) \oplus \mathcal{O}\left(\vec{x}_{2}-\vec{x}_{3}\right) \oplus$ $\mathcal{O}\left(\vec{x}_{3}-\vec{x}_{2}\right) \oplus E(-\vec{\omega})$. Under factoring line bundle summands in the distinguished exact sequence

$$
0 \rightarrow F \rightarrow E^{*} \oplus P\left(E\left\langle\vec{x}_{2}+\vec{x}_{3}\right\rangle\right) \rightarrow E\left\langle\vec{x}_{2}+\vec{x}_{3}\right\rangle \rightarrow 0
$$

we get a triangle

$$
E\left\langle\vec{x}_{2}+\vec{x}_{3}\right\rangle[-1] \rightarrow E(-\vec{\omega}) \rightarrow E^{*} \stackrel{\varphi}{\rightarrow} E\left\langle\vec{x}_{2}+\vec{x}_{3}\right\rangle .
$$

Comparing with (4.2), we get $E\left\langle\vec{x}_{2}+\vec{x}_{3}\right\rangle^{*}=E(-\vec{\omega})$, as claimed.

Therefore, we obtain a cluster tilting object in $\mathscr{C}$ with endomorphism algebra of the Gabriel quiver

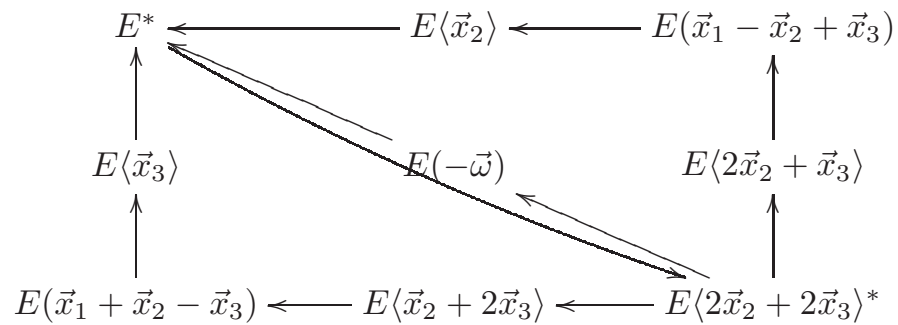

Let $T_{(2,4,4)}=\tau^{-1}\left(E^{*} \oplus E\left\langle\vec{x}_{2}\right\rangle \oplus E\left\langle\vec{x}_{3}\right\rangle \oplus E(-\vec{\omega})\right)[1] \oplus\left(E\left(\vec{x}_{1}-\vec{x}_{2}+\vec{x}_{3}\right) \oplus E\left(\vec{x}_{1}+\vec{x}_{2}-\right.\right.$ $\left.\left.\vec{x}_{3}\right) \oplus E\left\langle 2 \vec{x}_{2}+\vec{x}_{3}\right\rangle \oplus E\left\langle\vec{x}_{2}+2 \vec{x}_{3}\right\rangle \oplus E\left\langle 2 \vec{x}_{2}+2 \vec{x}_{3}\right\rangle^{*}\right)$. Then $T_{(2,4,4)}$ is a cluster tilting object in $\mathscr{C}$ with each direct summand belongs to the interval category $\mathscr{D}\left(\frac{2}{3}, \alpha\left(\frac{2}{3}\right)\right]$. Hence by Lemma 2.4, $T_{(2,4,4)}$ is a tilting object in $\mathscr{D}$ with endomorphism algebra:

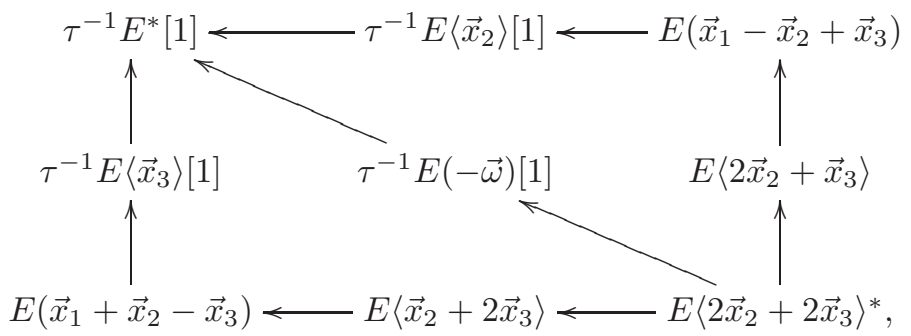

which is a tubular algebra of type $(2,4,4)$.

Next, we consider $\mathbb{X}$ of weight type $(2,3,6)$. Similarly, we start with the cluster tilting object $T_{\text {cub }}$ and the Gabriel quiver of its endomorphism algebra. It is more complicated than the weight type $(2,4,4)$ to obtain the desired tilting object by using the cluster mutation. We need use the mutations of the quiver at some vertex twice. We omit the process of describing the corresponding cluster tilting mutations to quiver mutations in the proof. 
Theorem 4.5. Assume that $\mathbb{X}$ has weight type $(2,3,6)$. Then there exists a tilting object $T_{(2,3,6)}$ in $\mathscr{D}$, whose endomorphism algebra is a tubular algebra of type $(2,3,6)$, here $T_{(2,3,6)}=\tau H[-1] \oplus E\left\langle 3 \vec{x}_{3}\right\rangle \oplus E\left(3 \vec{x}_{3}\right) \oplus E\left(2 \vec{x}_{2}-2 \vec{x}_{3}\right) \oplus E\left\langle 4 \vec{x}_{3}\right\rangle^{* *} \oplus$ $E\left\langle\vec{x}_{2}+\vec{x}_{3}\right\rangle \oplus E\left\langle\vec{x}_{2}\right\rangle\left(\vec{x}_{3}\right) \oplus \tau^{-1} E[1] \oplus \tau^{-1} E\left\langle\vec{x}_{3}\right\rangle[1] \oplus \tau^{-1} E\left\langle\vec{x}_{2}+2 \vec{x}_{3}\right\rangle^{*}[1]$ with the Gabriel quiver of the endomorphism algebra given as follows:

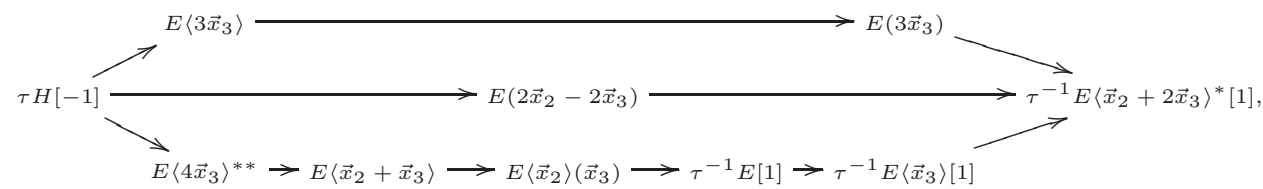

where $E\left\langle\vec{x}_{2}+2 \vec{x}_{3}\right\rangle^{*}$ is determined by the exact sequence

$$
0 \rightarrow E\left\langle\vec{x}_{3}\right\rangle \rightarrow E\left\langle\vec{x}_{2}+2 \vec{x}_{3}\right\rangle^{*} \rightarrow \mathcal{O}\left(\vec{x}_{2}+2 \vec{x}_{3}-\vec{x}_{1}\right) \rightarrow 0,
$$

$E\left\langle 4 \vec{x}_{3}\right\rangle^{* *}$ is determined by the exact sequence

$$
0 \rightarrow E\left\langle 2 \vec{x}_{3}\right\rangle(\vec{\omega}) \rightarrow E\left\langle 4 \vec{x}_{3}\right\rangle^{* *} \rightarrow \mathcal{O}\left(\vec{\omega}+\vec{x}_{2}\right) \rightarrow 0,
$$

$H$ is determined by the exact sequence

$$
0 \rightarrow E\left\langle 4 \vec{x}_{3}\right\rangle\left(\vec{x}_{2}\right) \rightarrow H \oplus \mathcal{O}\left(\vec{x}_{1}+\vec{x}_{2}+\vec{x}_{3}\right) \rightarrow G \rightarrow 0,
$$

and $G$ is determined by the exact sequence

$$
0 \rightarrow E\left\langle 2 \vec{x}_{3}\right\rangle\left(\vec{x}_{1}\right) \rightarrow G \rightarrow \mathcal{O}\left(\vec{x}_{1}+\vec{x}_{2}\right) \rightarrow 0 .
$$

Proof By Corollary 4.3, we get that $T^{\prime}=\tau^{-1} E[1] \oplus\left(\underset{0<\vec{x} \leq 2 \vec{\omega}+\vec{c}}{\bigoplus_{c}} E\langle\vec{x}\rangle\right)$ is a tilting object in $\mathscr{D}$, and each direct summand of $T^{\prime}$ belongs to $\mathscr{D}(0, \alpha(0)]$. Hence, by Lemma $2.4, T^{\prime}=T_{\text {cub }}$ in $\mathscr{C}$ is a cluster tilting object. The Gabriel quiver of the endomorphism algebra $\mathscr{C}\left(T^{\prime}, T^{\prime}\right)$ has the form:

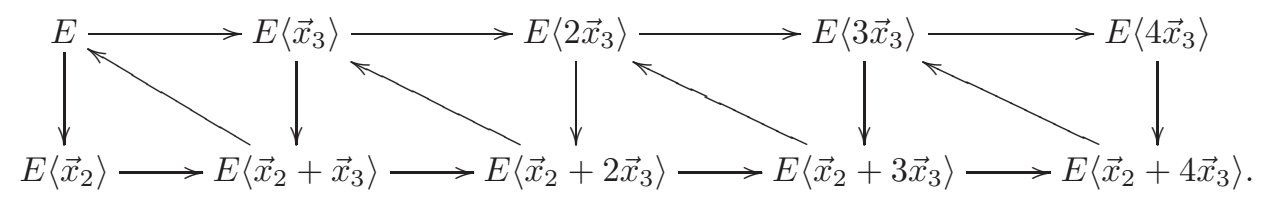

Using Keller's soft-ware, we know that under taking quiver mutations $u_{1} u_{2} u_{3} u_{4} u_{5} u_{6} u_{1}$ for the quiver

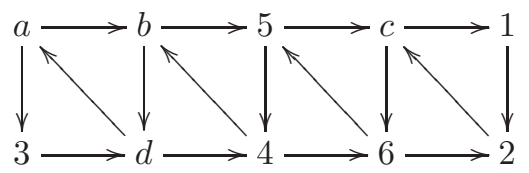

we can obtain the following quiver

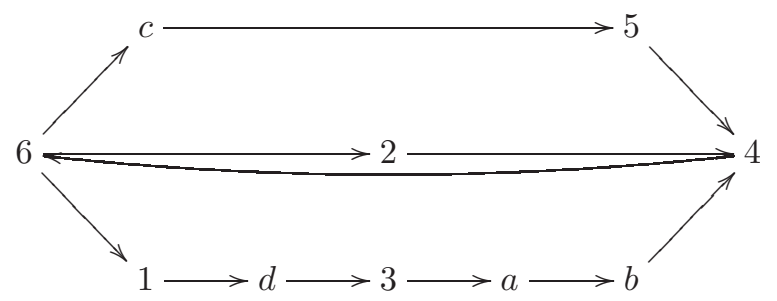


By using the relationship between the quiver mutation and the cluster tilting mutation, and the similar discussion to Theorem 4.4, we get that $T_{(2,3,6)}=\tau H[-1] \oplus$ $E\left\langle 3 \vec{x}_{3}\right\rangle \oplus E\left(3 \vec{x}_{3}\right) \oplus E\left(2 \vec{x}_{2}-2 \vec{x}_{3}\right) \oplus E\left\langle 4 \vec{x}_{3}\right\rangle^{* *} \oplus E\left\langle\vec{x}_{2}+\vec{x}_{3}\right\rangle \oplus E\left\langle\vec{x}_{2}\right\rangle\left(\vec{x}_{3}\right) \oplus \tau^{-1} E[1] \oplus$ $\tau^{-1} E\left\langle\vec{x}_{3}\right\rangle[1] \oplus \tau^{-1} E\left\langle\vec{x}_{2}+2 \vec{x}_{3}\right\rangle^{*}[1]$ is a cluster tilting object in $\mathscr{C}$, each of whose indecomposable direct summands belongs to $\mathscr{D}\left(\alpha^{-1}\left(\frac{11}{3}\right), \frac{11}{3}\right]$. Therefore $T_{(2,3,6)}$ is a tilting object in $\mathscr{D}$ with endomorphism algebra (4.5), which is a tubular algebra of type $(2,3,6)$.

In the rest of this section, we are devoted to the only left case, $\mathbb{X}$ of weight type $(3,3,3)$. Since $T_{\text {cub }}$ is not a cluster tilting object in the cluster category, we try to construct a replacement in $\mathscr{D}$ such that it is a cluster tilting object, then use the cluster mutation to get what we want.

Theorem 4.6. Assume that $\mathbb{X}$ has weight type $(3,3,3)$. Then there exists a tilting object $T_{(3,3,3)}=E \oplus\left(\bigoplus_{i=1}^{3} E\left\langle\vec{x}_{i}\right\rangle\right) \oplus\left(\bigoplus_{i=1}^{3} F_{i}[-1]\right) \oplus G$ in $\mathscr{D}$, whose endomorphism algebra is a tubular algebra of type $(3,3,3)$, here $F_{i}$ is determined by

$$
0 \rightarrow E\left\langle 2 \vec{x}_{i}\right\rangle \rightarrow F_{i} \rightarrow \mathcal{O}\left(\vec{x}_{1}+\vec{x}_{2}+\vec{x}_{3}\right) \rightarrow 0
$$

and $G$ is determined by the exact sequence for each $1 \leq i \leq 3$ :

$$
0 \rightarrow F_{i} \rightarrow G[1] \rightarrow E\left\langle\vec{x}_{1}+\vec{x}_{2}+\vec{x}_{3}\right\rangle\left(\vec{x}_{i}\right) \rightarrow 0 .
$$

Proof Firstly, we are going to find a tilting object in $\mathscr{D}$ such that each indecomposable summand belongs to the interval category $\mathscr{D}(a, \alpha(a)]$ for some $a \in \mathbb{Q}$.

By Proposition 3.2, there is a triangle in $\mathscr{D}$

$$
F_{i}[-1] \rightarrow E\left\langle\vec{x}_{i}+\vec{x}_{j}\right\rangle \oplus E\left\langle\vec{x}_{i}+\vec{x}_{k}\right\rangle \rightarrow E\left\langle\vec{x}_{1}+\vec{x}_{2}+\vec{x}_{3}\right\rangle \rightarrow F_{i},
$$

where $F_{i}$ is determined by the exact sequence

$$
0 \rightarrow E\left\langle 2 \vec{x}_{i}\right\rangle \rightarrow F_{i} \rightarrow \mathcal{O}\left(\vec{x}_{1}+\vec{x}_{2}+\vec{x}_{3}\right) \rightarrow 0 .
$$

Then from the following homotopy pullback commutative diagram induced by (4.9)

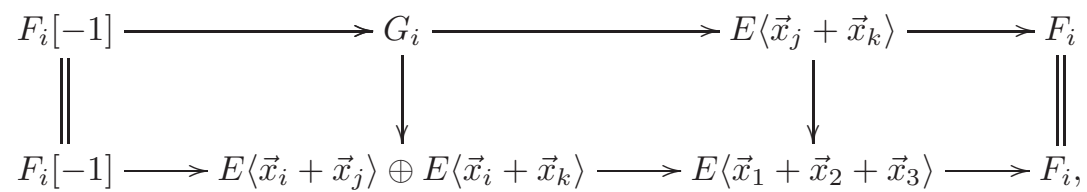

we get a triangle in $\mathscr{D}$

$$
G_{i} \rightarrow \bigoplus_{1 \leq t \neq l \leq 3} E\left\langle\vec{x}_{t}+\vec{x}_{l}\right\rangle \stackrel{\beta}{\rightarrow} E\left\langle\vec{x}_{1}+\vec{x}_{2}+\vec{x}_{3}\right\rangle \rightarrow G_{i}[1] .
$$

By the symmetry of the weights, we get that $\beta$ is independent of $i$, then also $G_{i}$, we denote it by $G$. Thus $G$ fits into the following triangle

$$
F_{i}[-1] \rightarrow G \rightarrow E\left\langle\vec{x}_{j}+\vec{x}_{k}\right\rangle \stackrel{\varphi_{i}}{\longrightarrow} F_{i} .
$$

Now we claim that $G$ is determined by (4.8). Without loss of generality, we assume that $i=2, j=1$ and $k=3$. Notice that the nonzero morphism $\varphi_{2}$ : $E\left\langle\vec{x}_{1}+\vec{x}_{3}\right\rangle \rightarrow F_{2}$ in $\mathscr{D}$ is injective in $\operatorname{coh} \mathbb{X}$, which fits into the following exact sequence

$$
0 \rightarrow E\left\langle\vec{x}_{1}+\vec{x}_{3}\right\rangle \stackrel{\varphi_{2}}{\longrightarrow} F_{2} \rightarrow \operatorname{cok} \varphi_{2} \rightarrow 0 .
$$


Since $E\left\langle\vec{x}_{1}+\vec{x}_{3}\right\rangle=E\left(\vec{c}-\vec{x}_{1}-\vec{x}_{3}\right)=E\left(\vec{\omega}+\vec{x}_{2}\right)$ and $\operatorname{Hom}\left(\mathcal{O}\left(2 \vec{\omega}+\vec{x}_{2}\right), F_{2}\right) \cong k$, we obtain the following commutative diagram

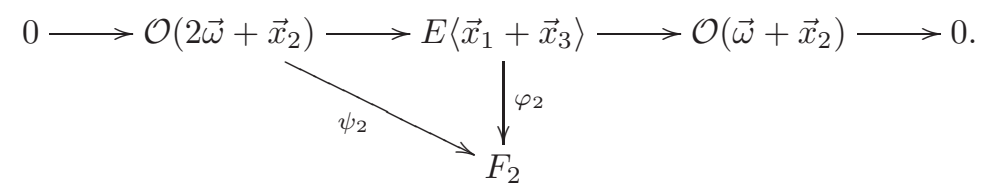

Then by the snake lemma, we get an exact sequence

$$
0 \rightarrow \mathcal{O}\left(\vec{\omega}+\vec{x}_{2}\right) \rightarrow \operatorname{cok} \psi_{2} \rightarrow \operatorname{cok} \varphi_{2} \rightarrow 0 .
$$

For calculation of $\operatorname{cok} \psi_{2}$, we consider the exact sequence

$$
0 \rightarrow \mathcal{O}\left(2 \vec{\omega}+\vec{x}_{2}\right) \stackrel{\psi_{2}}{\longrightarrow} F_{2} \rightarrow \operatorname{cok} \psi_{2} \rightarrow 0 .
$$

Notice that for any $\vec{x}>0, \operatorname{Hom}\left(\mathcal{O}\left(2 \vec{\omega}+\vec{x}_{2}+\vec{x}\right), F_{2}\right)=0$, we conclude that cok $\psi_{2} \in$ vect $\mathbb{X}$. Moreover, it is easy to obtain that $\left[\operatorname{cok} \psi_{2}\right]=\left[F_{2}\right]-\left[\mathcal{O}\left(2 \vec{\omega}+\vec{x}_{2}\right)\right]=\left[\mathcal{O}\left(2 \vec{x}_{1}+\right.\right.$ $\left.\left.\vec{x}_{2}\right)\right]+\left[\mathcal{O}\left(2 \vec{x}_{3}+\vec{x}_{2}\right)\right]$. It follows that $\operatorname{det}\left(\operatorname{cok} \psi_{2}\right)=2 \vec{x}_{1}+2 \vec{x}_{2}+2 \vec{x}_{3}$. If $\operatorname{cok} \psi_{2}$ is indecomposable, then $\mu\left(\operatorname{cok} \psi_{2}\right)=2$ implies that it is an Auslander bundle, that is, $\operatorname{cok} \psi_{2}=E(\vec{y})$ for some $\vec{y} \in \mathbb{L}$. Then $\operatorname{det}(E(\vec{y}))=\vec{\omega}+2 \vec{y}=2 \vec{x}_{1}+2 \vec{x}_{2}+2 \vec{x}_{3}$ implies that $\vec{y}=\vec{c}$. That is cok $\psi_{2}=E(\vec{c})$. But one can check that $[E(\vec{c})] \neq$ $\left[\mathcal{O}\left(2 \vec{x}_{1}+\vec{x}_{2}\right)\right]+\left[\mathcal{O}\left(2 \vec{x}_{3}+\vec{x}_{2}\right)\right]$, which is a contradiction. Hence, cok $\psi_{2}$ is a direct sum of two line bundles.

Recall that $I\left(E\left\langle 2 \vec{x}_{2}\right\rangle\right)=\mathcal{O}\left(2 \vec{x}_{2}\right) \oplus \mathcal{O}\left(\vec{x}_{2}+2 \vec{x}_{3}\right) \oplus \mathcal{O}\left(2 \vec{x}_{1}+\vec{x}_{2}\right) \oplus \mathcal{O}(\vec{\omega}+\vec{c})$ and all of the direct summands vanish under the functor $\operatorname{Ext}^{1}\left(\mathcal{O}\left(\vec{x}_{1}+\vec{x}_{2}+\vec{x}_{3}\right),-\right)$ except $\mathcal{O}\left(2 \vec{x}_{2}\right)$. Thus the exact sequence (4.10) induces the following commutative pushout diagram

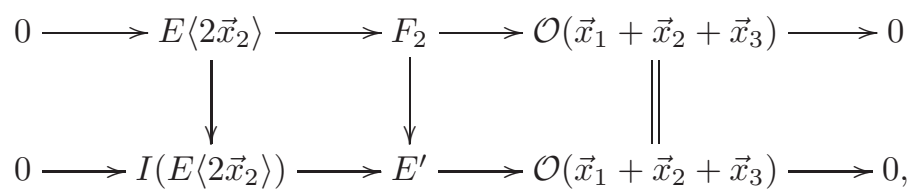

where $E^{\prime}=\left(I\left(E\left\langle 2 \vec{x}_{2}\right\rangle\right) \backslash \mathcal{O}\left(2 \vec{x}_{2}\right)\right) \oplus\left(E\left\langle\vec{x}_{2}\right\rangle\left(\vec{x}_{1}+\vec{x}_{3}\right)\right)$. Hence, we obtain a distinguished exact sequence

$$
0 \rightarrow E\left\langle 2 \vec{x}_{2}\right\rangle \rightarrow F_{2} \oplus \mathcal{O}\left(2 \vec{x}_{2}\right) \rightarrow E\left\langle\vec{x}_{2}\right\rangle\left(\vec{x}_{1}+\vec{x}_{3}\right) \rightarrow 0 .
$$

Thus we can easily show that the injective hull of $F_{2}$ has the expression $I\left(F_{2}\right)=$ $\mathcal{O}\left(\vec{x}_{2}+2 \vec{x}_{3}\right) \oplus \mathcal{O}\left(2 \vec{x}_{1}+\vec{x}_{2}\right) \oplus \mathcal{O}(\vec{\omega}+\vec{c}) \oplus \mathcal{O}\left(\vec{x}_{1}+\vec{x}_{2}+\vec{x}_{3}\right) \oplus \mathcal{O}\left(\vec{x}_{1}+2 \vec{x}_{2}\right) \oplus \mathcal{O}\left(2 \vec{x}_{2}+\vec{x}_{3}\right)$. By simple calculation, we find that only two direct summands of $I\left(F_{2}\right), \mathcal{O}\left(2 \vec{x}_{1}+\vec{x}_{2}\right)$ and $\mathcal{O}\left(\vec{x}_{2}+2 \vec{x}_{3}\right)$, satisfy that $\operatorname{det}\left(\mathcal{O}\left(2 \vec{x}_{1}+\vec{x}_{2}\right) \oplus \mathcal{O}\left(\vec{x}_{2}+2 \vec{x}_{3}\right)\right)=2 \vec{x}_{1}+2 \vec{x}_{2}+2 \vec{x}_{3}$. It follows that $\operatorname{cok} \psi_{2}=\mathcal{O}\left(2 \vec{x}_{1}+\vec{x}_{2}\right) \oplus \mathcal{O}\left(\vec{x}_{2}+2 \vec{x}_{3}\right)$. Hence $\operatorname{cok} \varphi_{2}$ fits into the following exact sequence

$$
0 \rightarrow \mathcal{O}\left(\vec{\omega}+\vec{x}_{2}\right) \rightarrow \mathcal{O}\left(2 \vec{x}_{1}+\vec{x}_{2}\right) \oplus \mathcal{O}\left(\vec{x}_{2}+2 \vec{x}_{3}\right) \rightarrow \operatorname{cok} \varphi_{2} \rightarrow 0 .
$$

Then the following pushout diagram

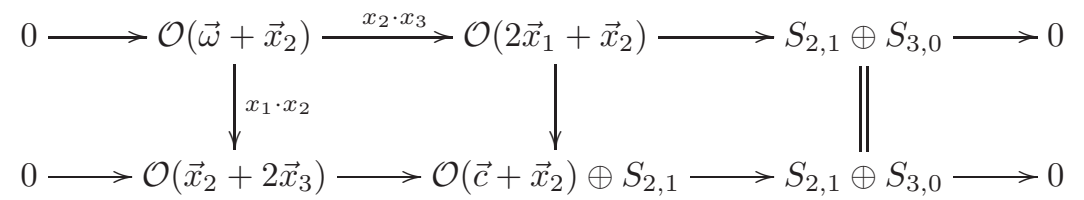


implies that $\operatorname{cok} \varphi_{2}=\mathcal{O}\left(\vec{c}+\vec{x}_{2}\right) \oplus S_{2,1}$.

Now we consider the following pushout diagram

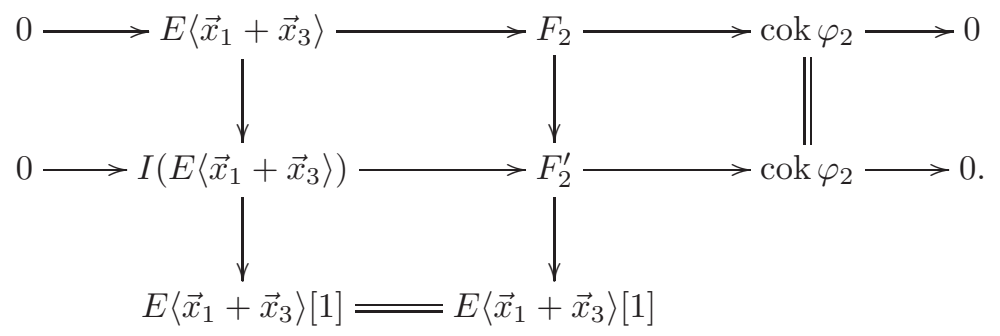

Since each direct summand of $I\left(E\left\langle\vec{x}_{1}+\vec{x}_{3}\right\rangle\right)$ does not vanish under the functor $\operatorname{Ext}^{1}\left(\operatorname{cok} \varphi_{2},-\right)$. Hence, $F_{2}^{\prime}$ contains no line bundle summands. Therefore, (4.22) induces a triangle in $\mathscr{D}$

$$
E\left\langle\vec{x}_{1}+\vec{x}_{3}\right\rangle \rightarrow F_{2} \rightarrow F_{2}^{\prime} \rightarrow E\left\langle\vec{x}_{1}+\vec{x}_{3}\right\rangle[1] .
$$

Comparing with (4.13), we obtain that $G=F_{2}^{\prime}[-1]$. Moreover, notice that $E\left\langle\vec{x}_{1}+\right.$ $\left.\vec{x}_{3}\right\rangle[1]=E\left\langle\vec{x}_{1}+\vec{x}_{2}+\vec{x}_{3}\right\rangle\left(\vec{x}_{2}\right)$, hence the fact that $\operatorname{Ext}^{1}\left(E\left\langle\vec{x}_{1}+\vec{x}_{2}+\vec{x}_{3}\right\rangle\left(\vec{x}_{2}\right), F_{2}\right) \cong k$ implies that $G$ is determined by the following distinguished exact sequence

$$
0 \rightarrow F_{2} \rightarrow G[1] \rightarrow E\left\langle\vec{x}_{1}+\vec{x}_{2}+\vec{x}_{3}\right\rangle\left(\vec{x}_{2}\right) \rightarrow 0 .
$$

This finishes the proof of the claim.

Since $\beta$ in (4.12) is the right (add $T_{\text {cub }} \backslash E\left\langle\vec{x}_{1}+\vec{x}_{2}+\vec{x}_{3}\right\rangle$ )-approximation of $E\left\langle\vec{x}_{1}+\right.$ $\left.\vec{x}_{2}+\vec{x}_{3}\right\rangle$. Hence by replacing $E\left\langle\vec{x}_{1}+\vec{x}_{2}+\vec{x}_{3}\right\rangle$ by $G$ in the tilting object $T_{\text {cub }}$, we get that $\bar{T}=\left(T_{\text {cub }} \backslash E\left\langle\vec{x}_{1}+\vec{x}_{2}+\vec{x}_{3}\right\rangle\right) \oplus G$ is a new tilting object in $\mathscr{D}$ such that each indecomposable direct summand belongs to $\mathscr{D}\left(\alpha^{-1}(1), 1\right]$. Moreover, the endomorphism algebra $\mathscr{D}(\bar{T}, \bar{T})$ has the shape

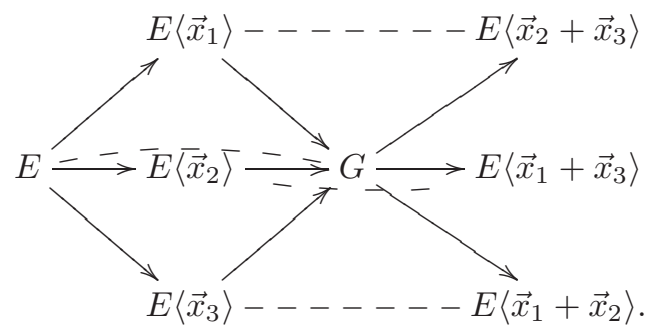

Then by Lemma $2.4, \bar{T}$ is a cluster tilting object in $\mathscr{C}$ such that $\mathscr{C}(\bar{T}, \bar{T})$ has the shape

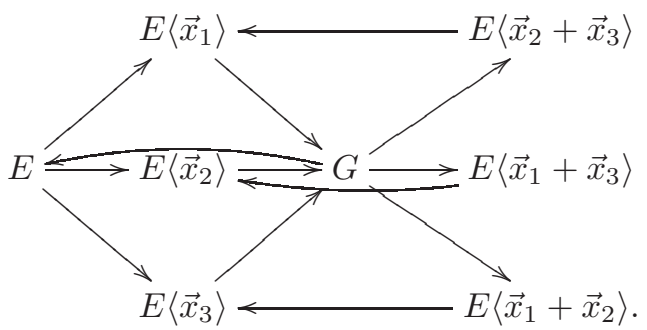


Using Keller's soft-ware, we find that by taking quiver mutations $u_{1} u_{2} u_{3}$ for the following quiver

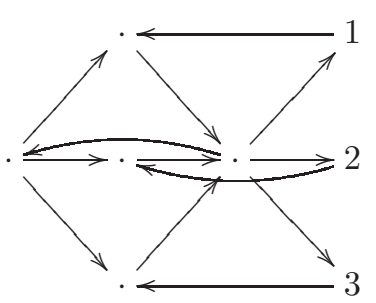

we obtain the quiver

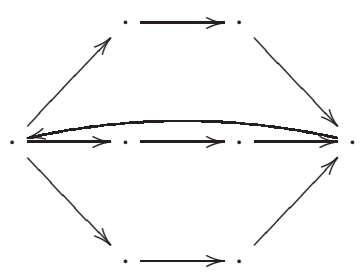

For $1 \leq i \leq 3$, the quiver mutation $u_{i}$ corresponds to the following cluster mutation

$$
E\left\langle\vec{x}_{j}+\vec{x}_{k}\right\rangle[-1] \rightarrow E\left\langle\vec{x}_{j}+\vec{x}_{k}\right\rangle^{*} \rightarrow G \rightarrow E\left\langle\vec{x}_{j}+\vec{x}_{k}\right\rangle,
$$

where $E\left\langle\vec{x}_{j}+\vec{x}_{k}\right\rangle^{*}=F_{i}[-1]$ by (4.13). Hence, $T_{(3,3,3)}=E \oplus\left(\bigoplus_{i=1}^{3} E\left\langle\vec{x}_{i}\right\rangle\right) \oplus$ $\left(\bigoplus_{i=1}^{3} F_{i}[-1]\right) \oplus G$ is a cluster tilting object in $\mathscr{C}$ with each indecomposable direct summand belongs to $\mathscr{D}\left(\alpha^{-1}(1), 1\right]$. Thus by Lemma $2.4, T_{(3,3,3)}$ is a tilting object in $\mathscr{D}$ with endomorphism algebra:

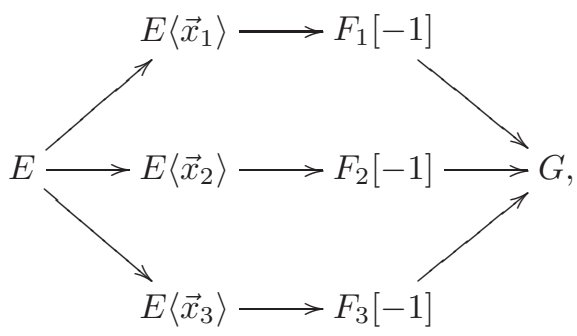

which is a tubular algebra of type $(3,3,3)$.

Acknowledgements. This work is partially supported by the National Natural Science Foundation of China (Grant Nos. 11571286, 11871404 and 11801473), the Natural Science Foundation of Fujian Province of China (Grant No. 2016J01031) and the Fundamental Research Funds for the Central Universities of China (Grant Nos. 20720180002 and 20720180006).

\section{REFERENCES}

1. M. Barot, D. Kussin and H. Lenzing, The cluster category of a canonical algebra. Trans. Amer. Math. Soc., 362 (2010), 4313-4330.

2. A. B. Buan, O. Iyama, I. Reiten and J. Scott, Cluster structures for 2-Calabi-Yau categories and unipotent groups. Compos. Math., 145(4) (2009), 1035-1079. 
3. A. B. Buan, R. Marsh, M. Reineke and I. Reiten and G. Todorov, Tilting theory and cluster combinatorics. Adv. Math., 204 (2006), 572-618.

4. J. Chen, Y. Lin and S. Ruan, Tilting objects in the stable category of vector bundles on the weighted projective line of type $(2,2,2,2 ; \lambda)$. J. Algebra., 397 (2014), 570-588.

5. J. Chen, Y. Lin, P. Liu and S. Ruan, Classifications for tilting objects on a weighted projective line of type $(2,2,2,2 ; \lambda)$. arXiv:1303.1323.

6. S. Fomin and A. Zelevinsky, Cluster algebras I. Foundations. J. Amer. Math. Soc., 15(2) (2002), 497-529.

7. W. Geigle and $\mathrm{H}$. Lenzing, A class of weighted projective curves arising in representation theory of finite dimensional algebras. Singularities, representations of algebras, and Vector bundles, Springer Lect. Notes Math., 1273 (1987), 265-297.

8. D. Happel, Triangulated categories in the representation theory of finite-dimensional algebras. London Mathematical Society Lecture Note Series, Cambridge University Press, Cambridge, 119 (1988).

9. O. Iyama and Y. Yoshino, Mutation in triangulated categories and rigid Cohen-Macaulay modules. Invent. Math., 172 (2008), 117-168.

10. B. Keller, On triangulated orbit categories. Doc. Math., 10 (2005), 551-581.

11. B. Keller and I. Reiten, Acyclic Calabi-Yau categories. Compos. Math., 144 (5) (2008), 1332-1348.

12. D. Kussin, H. Lenzing and H. Meltzer, Triangle singularities, ADE-chains and weighted projective lines. Adv. Math., 237(1) (2013), 194-251.

13. H. Lenzing, Wild canonical algebras and rings of automorphic forms. In Finite-dimensional algebras and related topics (Ottawa, ON, 1992), volume 424 of NATO Adv. Sci. Inst. Ser. C Math. Phys. Sci., 191-212. Kluwer Acad. Publ., Dordrecht, 1994.

14. H. Lenzing and H. Meltzer, Sheaves on a weighted projective line of genus one, and representations of a tubular algebra. In Representations of algebras, Sixth International Conference, Ottawa 1992. CMS Conf. Proc., 14 (1993), 313-337.

15. H. Lenzing and S. Ruan, On vector bundles of rank two on a weighted projective line. In Preparation.

16. D. Orlov, Derived categories of coherent sheaves and triangulated categories of singularities. Algebra, Arithmetic, and Geometry, Progress in Mathematics, 270 (2009), 503-531.

School of Mathematical Sciences, Xiamen University, Xiamen 361005, P.R.China

E-mail address: chenjianmin@xmu.edu.cn

School of Mathematical Sciences, Xiamen University, Xiamen 361005, P.R.China

E-mail address: ynlin@xmu.edu.cn

School of Mathematical Sciences, Xiamen University, Xiamen 361005, P.R.China

E-mail address: sqruan@xmu.edu.cn 